\title{
PENALIZATION OF DIRICHLET OPTIMAL CONTROL PROBLEMS*
}

\author{
Eduardo Casas ${ }^{1}$, Mariano Mateos $^{2}$ and Jean-Pierre Raymond ${ }^{3}$
}

\begin{abstract}
We apply Robin penalization to Dirichlet optimal control problems governed by semilinear elliptic equations. Error estimates in terms of the penalization parameter are stated. The results are compared with some previous ones in the literature and are checked by a numerical experiment. A detailed study of the regularity of the solutions of the PDEs is carried out.
\end{abstract}

Mathematics Subject Classification. 49M30, 35B30, 35B37.

Received July 4, 2007. Revised May 6, 2008.

Published online August 20, 2008.

\section{INTRODUCTION}

The purpose of this paper is to study solution strategies for the control problem

$$
(\mathrm{P})\left\{\begin{array}{l}
\operatorname{Min} J(u)=\int_{\Omega} L\left(x, y_{u}(x)\right) \mathrm{d} x+\frac{N}{2} \int_{\Gamma} u(x)^{2} \mathrm{~d} \sigma(x) \\
u \in U_{a d}=\left\{u \in L^{2}(\Gamma): \alpha \leq u(x) \leq \beta \text { a.e. } x \in \Gamma\right\}, \\
-\Delta y_{u}+a\left(x, y_{u}\right)=0 \text { in } \Omega, y_{u}=u \text { on } \Gamma,
\end{array}\right.
$$

where $\Omega$ is an open convex bounded polygonal domain in $\mathbb{R}^{2}, \Gamma$ is its boundary, $N>0,-\infty<\alpha<\beta<+\infty$ and $a(x, y)$ and $L(x, y)$ are functions whose properties will be stated later. The case when $\alpha=-\infty$ and $\beta=\infty$ is studied for linear equations in Section 7. We approximate problem (P) by

$$
\left(\mathrm{P}_{\varepsilon}\right)\left\{\begin{array}{l}
\operatorname{Min} J_{\varepsilon}(u)=\int_{\Omega} L\left(x, y_{\varepsilon, u}(x)\right) \mathrm{d} x+\frac{N}{2} \int_{\Gamma} u(x)^{2} \mathrm{~d} \sigma(x) \\
u \in U_{a d}, \\
-\Delta y_{\varepsilon, u}+a\left(x, y_{\varepsilon, u}\right)=0 \text { in } \Omega, \varepsilon \partial_{\nu} y_{\varepsilon, u}+y_{\varepsilon, u}=u \text { on } \Gamma,
\end{array}\right.
$$

with $\varepsilon>0$ small enough.

\footnotetext{
Keywords and phrases. Dirichlet optimal control, Robin penalization, regularity of solutions.

* The first two authors were partially supported by Spanish Ministry of Education and Science under projects MTM2005-06817 and "Ingenio Mathematica (i-MATH)" No. CSD2006-00032 (Consolider Ingenio 2010).

${ }^{1}$ Dpto. de Matemática Aplicada y Ciencias de la Computación, E.T.S.I. Industriales y de Telecomunicación, Universidad de Cantabria, 39005 Santander, Spain. eduardo.casas@unican.es

2 Dpto. de Matemáticas, Universidad de Oviedo, Campus de Viesques, 33203 Gijón, Spain. mmateos@uniovi.es

3 Institut de Mathématiques de Toulouse, UMR CNRS 5219, Université Paul Sabatier, 31062 Toulouse Cedex 9, France. raymond@mip.ups-tlse.fr
} 
The main objective of our paper is to show that local solutions $\bar{u}$ to problem (P) satisfying a sufficient second order optimality condition may be approximated by a sequence $\left\{\bar{u}_{\varepsilon}\right\}$ of local solutions to $\left(\mathrm{P}_{\varepsilon}\right)$, and that

$$
\left\|\bar{u}-\bar{u}_{\varepsilon}\right\|_{L^{2}(\Gamma)} \leq C \varepsilon^{1-1 / p}
$$

for some $p>2$.

Approximations of Dirichlet control problems by penalized Robin control problems have been studied in [12] in the case of the stationary Navier-Stokes equations and in [2] in the case of linear elliptic equations without control constraints. A convergence result is stated in [12] without error estimates in function of $\varepsilon$. In [2], error estimates of order $\varepsilon^{1 / 2}$ are proved for regular domains, and of order $\varepsilon^{1 / 2-\delta}$ for all $\delta>0$ in the case of convex polygons. Also mention that the case of parabolic equations is studied in [3].

By establishing an error of order $\varepsilon^{1-1 / p}$ where $p$ is precisely defined in function of the angles of the polygons and the data of the problems, we clearly improve the results of [2] (see also Sect. 7).

A key point in the proof is based on regularity results for the normal derivative of solutions to elliptic equations that we state in Lemma A.2. We think that such results are new and can be useful in other situations.

We present a detailed numerical experiment which shows that our theory is quite sharp. Throughout all the paper we make intensive use of regularity results for elliptic PDEs and some nonstandard trace results in polygons. They are all collected and proved in detail in an appendix.

\section{Assumptions And main RESUlts}

We will make the following assumptions on the functions that appear in the nonlinear term of the state equation and in the functional.

(A1) $a: \Omega \times \mathbb{R} \rightarrow \mathbb{R}$ is measurable with respect to the first variable and of class $C^{2}$ with respect to the second one, $a(x, 0) \in L^{\bar{p}}(\Omega), \bar{p}>2, \partial_{y} a(x, y) \geq 0$ for a.e. $x \in \Omega$ and every $y \in \mathbb{R}$. For all $M>0$ there exists a constant $C_{a, M}$ such that

$$
\begin{gathered}
\left|\partial_{y} a(x, y)\right|+\left|\partial_{y y}^{2} a(x, y)\right| \leq C_{a, M} \text { for a.e. } x \in \Omega \text { and }|y| \leq M, \\
\left|\partial_{y y}^{2} a\left(x, y_{1}\right)-\partial_{y y}^{2} a\left(x, y_{2}\right)\right|<C_{a, M}\left|y_{2}-y_{1}\right| \text { for a.e. } x \in \Omega \text { and }\left|y_{1}\right|,\left|y_{2}\right| \leq M .
\end{gathered}
$$

(A2) $L: \Omega \times \mathbb{R} \rightarrow \mathbb{R}$ is measurable with respect to the first variable and of class $C^{2}$ with respect to the second one, $L(x, 0) \in L^{1}(\Omega)$. For all $M>0$ there exist a constant $C_{L, M}$ and a function $\Psi_{L, M} \in L^{\bar{p}}(\Omega)$ such that

$$
\begin{gathered}
\left|\partial_{y} L(x, y)\right| \leq \Psi_{L, M}(x),\left|\partial_{y y}^{2} L(x, y)\right| \leq C_{L, M} \text { for a.e. } x \in \Omega \text { and }|y| \leq M \\
\left|\partial_{y y}^{2} L\left(x, y_{1}\right)-\partial_{y y}^{2} L\left(x, y_{2}\right)\right|<C_{L, M}\left|y_{2}-y_{1}\right| \text { for a.e. } x \in \Omega \text { and }\left|y_{1}\right|,\left|y_{2}\right| \leq M
\end{gathered}
$$

Let $\omega$ be the biggest angle of the convex polygonal domain $\Omega$ and define $p_{\Omega}=2 /(2-\pi / \omega)$ if $\omega>\pi / 2$. In the rest of the paper the exponent $p$ is defined by

$$
p=\left\{\begin{array}{cl}
\min \left\{\bar{p}, p_{\Omega}\right\} & \text { if } \omega>\pi / 2 \\
\bar{p} & \text { if } \omega \leq \pi / 2 \text { and } \bar{p}<+\infty
\end{array}\right.
$$

If $\omega \leq \pi / 2$ and $\bar{p}=\infty$, then $p$ can be arbitrarily chosen in the interval $(2,+\infty)$.

Let us now state the main result of the paper.

Theorem 2.1. Suppose that $\bar{u}$ is a local solution of $(\mathrm{P})$ satisfying the second order sufficient optimality conditions (6.4). Then there exist $\rho>0$ and $\varepsilon^{*}>0$ such that, for all $0<\varepsilon<\varepsilon^{*},\left(\mathrm{P}_{\varepsilon}\right)$ has at least a local solution $\bar{u}_{\varepsilon}$ in $B_{\rho}(\bar{u}) \cap U_{a d}=\left\{u \in U_{a d}:\|u-\bar{u}\|_{L^{\infty}(\Gamma)}<\rho\right\}$, and any sequence $\left\{\bar{u}_{\varepsilon}\right\}$ of local solutions of $\left(\mathrm{P}_{\varepsilon}\right)$ in $B_{\rho}(\bar{u}) \cap U_{a d}$ obeys

$$
\left\|\bar{u}-\bar{u}_{\varepsilon}\right\|_{L^{2}(\Gamma)} \leq C \varepsilon^{1-1 / p}
$$


Moreover $\bar{u}$ belongs to $C(\Gamma)$, and the sequences $\left\{\bar{u}_{\varepsilon}\right\}$ of local solutions to $\left(\mathrm{P}_{\varepsilon}\right)$ in $B_{\rho}(\bar{u}) \cap U_{\text {ad }}$ converges to $\bar{u}$ in $C(\Gamma)$.

To prove this result, we have to study the following linear equation

$$
-\Delta y_{\varepsilon}+a_{0}(x) y_{\varepsilon}=0 \text { in } \Omega, \varepsilon \partial_{\nu} y_{\varepsilon}+y_{\varepsilon}=u \text { on } \Gamma,
$$

where $a_{0} \in L^{p}(\Omega), a_{0} \geq 0$ for a.e. $x \in \Omega$. In the following theorem and in Appendix A, $C(M)$ will denote different constants depending on $M$, where $\left\|a_{0}\right\|_{L^{p}(\Omega)} \leq M$.

Theorem 2.2. Let $u \in H^{s}(\Gamma)$, with $-1 \leq s \leq 1$. The unique solution $y_{\varepsilon} \in H^{\min \{2, s+3 / 2\}}(\Omega)$ of equation (2.1) obeys

$$
\left\|y_{\varepsilon}\right\|_{H^{t}(\Gamma)} \leq C(M) \varepsilon^{s-t}\|u\|_{H^{s}(\Gamma)}
$$

for all $s \leq t \leq \min \{1, s+1\}$. Moreover, for all $-\frac{1}{2} \leq s \leq 1, s \leq t \leq \min \{1, s+1\}$

$$
\left\|y_{\varepsilon}\right\|_{H^{t+1 / 2}(\Omega)} \leq C(M) \varepsilon^{s-t}\|u\|_{H^{s}(\Gamma)} .
$$

Remark 2.3. The constant $C(M)$ that appears in Theorem 2.2 depends only on an upper bound of the norm of $a_{0}$ in $L^{p}(\Omega)$ and not on $a_{0}$ itself, as is carefully shown in all the proofs of Appendix A. So this result can be applied to the solutions of

$$
-\Delta y_{\varepsilon}+a_{\varepsilon}(x) y_{\varepsilon}=0 \text { in } \Omega, \varepsilon \partial_{\nu} y_{\varepsilon}+y_{\varepsilon}=u \text { on } \Gamma,
$$

where $\left\{a_{\varepsilon}: 0<\varepsilon<1\right\}$ is a family of nonnegative functions uniformly bounded in $L^{p}(\Omega)$.

A similar result appears in [9] in the case of regular domains and when $a_{0}(x)=0$. The proof of this result, as well as the precise meaning of solutions to equations when the data are not regular are stated in Appendix A.

The rest of the paper is as follows. In the next section we study the state equations for $(P)$ and $\left(P_{\varepsilon}\right)$, and we estimate the difference between the state and the penalized state for a fixed control. In Section 4, we recall the well known first order optimality conditions and some of the properties of the solutions that we can deduce from them. In Section 5, we prove the convergence of solutions of the penalized problems to the solution of the original problem. Next, we estimate the difference between the normal derivatives of the adjoint states of the original and of the penalized problems. In Section 6, we introduce second order sufficient optimality conditions and we prove Theorem 2.1. In Section 7, we explain how to apply our method to unconstrained linear quadratic problems. In Section 8, some numerical experiments are reported. The proof of Theorem 2.2 is given in Appendix A.

\section{State Equation}

We have the following results concerning the state equation.

Lemma $3.1([5])$. For all $u \in L^{\infty}(\Gamma) \cap H^{\sigma}(\Gamma)$, with $0 \leq \sigma \leq 1 / 2$, the state equation

$$
-\Delta y_{u}+a\left(x, y_{u}\right)=0 \text { in } \Omega, y_{u}=u \text { on } \Gamma
$$

has a unique solution, $y_{u} \in L^{\infty}(\Omega) \cap H^{1 / 2+\sigma}(\Omega)$ and it satisfies the estimate

$$
\left\|y_{u}\right\|_{L^{\infty}(\Omega)} \leq\|u\|_{L^{\infty}(\Gamma)}+C\|a(\cdot, 0)\|_{L^{p}(\Omega)} .
$$

Moreover, the following Lipschitz properties hold

$$
\left\|y_{u}-y_{v}\right\|_{L^{\infty}(\Omega)} \leq\|u-v\|_{L^{\infty}(\Gamma)},\left\|y_{u}-y_{v}\right\|_{H^{1 / 2+\sigma}(\Omega)} \leq C\|u-v\|_{H^{\sigma}(\Gamma)}
$$

for all $u, v \in L^{\infty}(\Gamma) \cap H^{\sigma}(\Gamma)$. Finally if $u_{n} \rightarrow u$ weakly* in $L^{\infty}(\Gamma)$, then $y_{u_{n}} \rightarrow y_{u}$ strongly in $L^{r}(\Omega)$ for all $r<+\infty$. 
Lemma 3.2. For all $u \in L^{\infty}(\Gamma) \cap H^{\sigma}(\Gamma)$, with $0 \leq \sigma \leq 1 / 2$, there exists a unique $y_{\varepsilon, u} \in H^{3 / 2+\sigma}(\Omega)$ solution of

$$
-\Delta y_{\varepsilon, u}+a\left(x, y_{\varepsilon, u}\right)=0 \text { in } \Omega, \varepsilon \partial_{\nu} y_{\varepsilon, u}+y_{\varepsilon, u}=u \text { on } \Gamma \text {. }
$$

Moreover

where $C$ is independent of $\varepsilon$.

$$
\left\|y_{\varepsilon, u}\right\|_{L^{\infty}(\Omega)} \leq\|u\|_{L^{\infty}(\Gamma)}+C\|a(\cdot, 0)\|_{L^{p}(\Omega)},
$$

Proof. The existence of a unique solution in $H^{1}(\Omega) \cap L^{\infty}(\Omega)$ to equation (3.2) is already proved in [1], Theorem 5 . To establish the more precise estimate (3.3), notice that $y_{\varepsilon, u}=y_{0}+y_{1}$, where $y_{0}$ is the solution of

$$
-\Delta y_{0}+\int_{0}^{1} \partial_{y} a\left(x, \theta y_{\varepsilon, u}\right) \mathrm{d} \theta y_{0}=-a(x, 0) \text { in } \Omega, \quad \varepsilon \partial_{\nu} y_{0}+y_{0}=0 \text { on } \Gamma
$$

and $y_{1}$ is the solution of

$$
-\Delta y_{1}+\int_{0}^{1} \partial_{y} a\left(x, \theta y_{\varepsilon, u}\right) \mathrm{d} \theta y_{1}=0 \text { in } \Omega, \quad \varepsilon \partial_{\nu} y_{1}+y_{1}=u \text { on } \Gamma .
$$

We can easily show that

$$
\left\|y_{1}\right\|_{L^{\infty}(\Omega)} \leq\|u\|_{L^{\infty}(\Gamma)}
$$

by observing that $\pm\|u\|_{L^{\infty}(\Gamma)}$ are upper and lower solutions to the equation satisfied by $y_{1}$ (see $e . g$. [15] for the notions of upper and lower solutions). The estimate

$$
\left\|y_{0}\right\|_{L^{\infty}(\Omega)} \leq C\|a(\cdot, 0)\|_{L^{p}(\Omega)}
$$

follows from Theorem 2 in [1]. Thus the $L^{\infty}(\Omega)$-estimate is proved.

To deduce the regularity $y_{\varepsilon, u} \in H^{3 / 2+\sigma}(\Omega)$ we use assumption (A1) and Lemma A.5 in the appendix, taking into account that we can write the boundary condition

$$
\varepsilon \partial_{\nu} y_{\varepsilon, u}+y_{\varepsilon, u}=u \text { on } \Gamma
$$

equivalently as

$$
\partial_{\nu} y_{\varepsilon, u}+y_{\varepsilon, u}=\frac{u}{\varepsilon}+\frac{\varepsilon-1}{\varepsilon} y_{\varepsilon, u} \text { on } \Gamma .
$$

Since we know that $y_{\varepsilon, u} \in H^{1}(\Omega)$, then $\left.y_{\varepsilon, u}\right|_{\Gamma} \in H^{1 / 2}(\Gamma)$ and the result follows from Lemma A.5 for $s=\sigma$.

Remark 3.3. Lemmas 3.1 and 3.2 imply the existence of $K>0$ depending on $\alpha, \beta$ and $\|a(\cdot, 0)\|_{L^{p}(\Omega)}$, such that for all $u \in U_{a d}$,

$$
\left\|y_{\varepsilon, u}\right\|_{L^{\infty}(\Omega)} \leq K,\left\|y_{u}\right\|_{L^{\infty}(\Omega)} \leq K
$$

Throughout the following, we often use estimates for the normal derivative of solutions to elliptic equations which are not standard and that we prove in Appendix A. Let us state these results. In Lemma A.6, we prove that the solution to equation

obeys

$$
-\Delta y+a_{0}(x) y=0 \text { in } \Omega, y=\eta \text { on } \Gamma
$$

$$
\left\|\partial_{\nu} y\right\|_{H^{s-1}(\Gamma)} \leq C(M)\|\eta\|_{H^{s}(\Gamma)}
$$

when $0 \leq s \leq 1$. Here $\left\|a_{0}\right\|_{L^{p}(\Omega)} \leq M, C(M)$ is a constant depending on $M$, and the solution $y$ together with $\partial_{\nu} y$ are defined in the sense of transposition.

In Lemma A.2, we prove that the solution to equation

$$
-\Delta z+a_{0}(x) z=g \text { in } \Omega, \quad z=0 \text { on } \Gamma,
$$


obeys

$$
\left\|\partial_{\nu} z\right\|_{W^{1-1 / q, q(\Gamma)}} \leq C(M)\|g\|_{L^{q}(\Omega)} .
$$

Now, for a fixed control, we can estimate the difference between the state and the approximate state.

Theorem 3.4. Let $u$ belong to $L^{\infty}(\Gamma) \cap H^{1 / 2}(\Gamma)$. Let $y_{u}$ be the solution of (3.1) and $y_{\varepsilon, u}$ be the solution of (3.2). Then there exist $C>0$ and $\varepsilon_{0}>0$ such that for all $0<\varepsilon<\varepsilon_{0}$

$$
\left\|y_{u}-y_{\varepsilon, u}\right\|_{L^{2}(\Omega)} \leq C \varepsilon
$$

where $C$ is a constant depending on $\|a(\cdot, 0)\|_{L^{2}(\Omega)}$, and on $R$ (an upper bound for $\left.\|u\|_{L^{\infty}(\Gamma)}+\|u\|_{H^{1 / 2}(\Gamma)}\right)$, but is independent of $u$, provided that $\|u\|_{L^{\infty}(\Gamma)}+\|u\|_{H^{1 / 2}(\Gamma)} \leq R$.

Proof. The function $y_{\varepsilon, u}-y_{u}$ obeys the equation

$$
\begin{gathered}
-\Delta\left(y_{\varepsilon, u}-y_{u}\right)+\int_{0}^{1} \partial_{y} a\left(x, y_{u}+\theta\left(y_{\varepsilon, u}-y_{u}\right)\right) \mathrm{d} \theta\left(y_{\varepsilon, u}-y_{u}\right)=0 \text { in } \Omega, \\
\varepsilon \partial_{\nu}\left(y_{\varepsilon, u}-y_{u}\right)+\left(y_{\varepsilon, u}-y_{u}\right)=-\varepsilon \partial_{\nu} y_{u} \text { on } \Gamma .
\end{gathered}
$$

Assumption (A1), the uniform boundness of $\left\|y_{\varepsilon, u}\right\|_{L^{\infty}(\Omega)}$, and Remark 2.3 allow us to use Theorem 2.2 with $s=t=-1 / 2$ to obtain

$$
\left\|y_{\varepsilon, u}-y_{u}\right\|_{L^{2}(\Omega)} \leq C \varepsilon\left\|\partial_{\nu} y_{u}\right\|_{H^{-1 / 2}(\Gamma)}
$$

To estimate $\partial_{\nu} y_{u}$, we observe that $y_{u}=z_{u}+\zeta$, where $z_{u}$ obeys $\Delta z_{u}=0$ in $\Omega$ and $z_{u}=u$ on $\Gamma$, and $\Delta \zeta=-a\left(x, y_{u}\right)$ in $\Omega$ and $\zeta=0$ on $\Gamma$. From estimate (3.4) for $s=1 / 2$, we get

$$
\left\|\partial_{\nu} z_{u}\right\|_{H^{-1 / 2}(\Gamma)} \leq C\|u\|_{H^{1 / 2}(\Gamma)} .
$$

With estimate (3.5), we can write

$$
\left\|\partial_{\nu} \zeta\right\|_{H^{1 / 2}(\Gamma)} \leq C\left\|a\left(\cdot, y_{u}\right)\right\|_{L^{2}(\Omega)} \leq C\left(\|a(\cdot, 0)\|_{L^{2}(\Omega)}+\kappa C_{a, \kappa}\right),
$$

where $\left\|y_{u}\right\|_{L^{\infty}(\Omega)} \leq \kappa$.

\section{First order optimality CONDitions}

The existence of at least one solution to problem (P) can be proved in a classical way. The optimality conditions for our problems are well known in the literature. We summarize them, as well as some of their immediate consequences in the following lemmas. For detailed proofs we refer to $[5,7]$. For every $u \in U_{a d}$, we define the adjoint state associated with $u$ as the unique solution to equation

$$
-\Delta \varphi_{u}+\partial_{y} a\left(x, y_{u}(x)\right) \varphi_{u}=\partial_{y} L\left(x, y_{u}(x)\right) \text { in } \Omega, \quad \varphi_{u}=0 \text { on } \Gamma .
$$

Lemma 4.1 ([5]). Let $\bar{u} \in L^{\infty}(\Gamma)$ be a solution of $(\mathrm{P})$, and let $\bar{y}=y_{\bar{u}} \in H^{1 / 2}(\Omega) \cap L^{\infty}(\Omega)$ be its associated state. Then $\bar{\varphi}=\varphi_{\bar{u}}$, the adjoint state associated to $\bar{u}$, belongs to $W^{2, p}(\Omega)$ and

$$
\bar{u}(x)=\operatorname{Proj}_{[\alpha, \beta]}\left(\frac{1}{N} \partial_{\nu} \bar{\varphi}(x)\right) .
$$

Moreover, $\bar{u}$ belongs to $W^{1-1 / p, p}(\Gamma)$ and $\bar{y} \in W^{1, p}(\Omega)$.

In a similar way, for every $u \in U_{a d}$ we define the approximate adjoint state $\varphi_{\varepsilon, u}$ associated with $u$ as the unique solution to equation

$$
-\Delta \varphi_{\varepsilon, u}+\partial_{y} a\left(x, y_{\varepsilon, u}(x)\right) \varphi_{\varepsilon, u}=\partial_{y} L\left(x, y_{\varepsilon, u}(x)\right) \text { in } \Omega, \quad \varepsilon \partial_{\nu} \varphi_{\varepsilon, u}+\varphi_{\varepsilon, u}=0 \text { on } \Gamma .
$$


Lemma $4.2([7])$. For $0<\varepsilon<1$, let $\bar{u}_{\varepsilon} \in L^{\infty}(\Gamma)$ be a solution of $\left(\mathrm{P}_{\varepsilon}\right)$, and let $\bar{y}_{\varepsilon}=y_{\varepsilon, \bar{u}_{\varepsilon}} \in W^{2, p}(\Omega)$ be its associated approximate state. Then $\bar{\varphi}_{\varepsilon}=\varphi_{\varepsilon, \bar{u}_{\varepsilon}}$, the approximate adjoint state associated with $\bar{u}_{\varepsilon}$, belongs to $W^{2, p}(\Omega)$ and

$$
\bar{u}_{\varepsilon}(x)=\operatorname{Proj}_{[\alpha, \beta]}\left(\frac{-1}{N \varepsilon} \bar{\varphi}_{\varepsilon}(x)\right)=\operatorname{Proj}_{[\alpha, \beta]}\left(\frac{1}{N} \partial_{\nu} \bar{\varphi}_{\varepsilon}(x)\right) .
$$

Remark 4.3. From the first equality in equation (4.3), we can deduce that $\bar{u}_{\varepsilon} \in C^{0,1}(\Gamma)$. Nevertheless, the Lipschitz constant of $\bar{u}_{\varepsilon}$ may depend on $1 / \varepsilon$. Actually, we will only use the fact that $\bar{u}_{\varepsilon} \in H^{1-1 / p}(\Gamma)$, which will be proved in Proposition 5.3 below.

\section{Convergence And Study of the ADjoint EQUATion}

First, we are going to show that the approximate optimal controls are bounded, not only in $L^{\infty}(\Gamma)$, but in an appropriate Sobolev space, and we will be able to deduce the uniform convergence of sequences of solutions of the approximate control problems. To do this, we introduce a kind of "intermediate" adjoint state between $\varphi_{u}$ and $\varphi_{\varepsilon, u}$. For every $u \in L^{\infty}(\Gamma)$, let us define $\psi_{\varepsilon, u}$ the solution of

$$
-\Delta \psi_{\varepsilon, u}+\partial_{y} a\left(x, y_{\varepsilon, u}\right) \psi_{\varepsilon, u}=\partial_{y} L\left(x, y_{\varepsilon, u}\right) \text { in } \Omega, \psi_{\varepsilon, u}=0 \text { on } \Gamma
$$

Lemma 5.1. There exists $C>0$ such that for every $u \in U_{\text {ad }}$ and every $0<\varepsilon<1$

and

$$
\begin{gathered}
\left\|\psi_{\varepsilon, u}\right\|_{L^{\infty}(\Omega)} \leq C, \\
\left\|\partial_{\nu} \psi_{\varepsilon, u}\right\|_{H^{1-1 / p}(\Gamma)} \leq C,
\end{gathered}
$$

$$
\left\|\partial_{\nu} \varphi_{\varepsilon, u}\right\|_{H^{1-1 / p}(\Gamma)} \leq C .
$$

Proof. Assumptions (A1) and (A2) and Remark 3.3 imply

$$
\left\|\partial_{y} a\left(\cdot, y_{\varepsilon, u}(\cdot)\right)\right\|_{L^{\infty}(\Omega)} \leq C_{a, K},\left\|\partial_{y} L\left(\cdot, y_{\varepsilon, u}(\cdot)\right)\right\|_{L^{p}(\Omega)} \leq\left\|\Psi_{L, K}\right\|_{L^{p}(\Omega)}
$$

for all $0<\varepsilon<1$, where $K$ is the constant introduced in Remark 3.3. Estimate (5.2) can now be deduced from [17].

Using estimate (3.5), we deduce that

$$
\left\|\partial_{\nu} \psi_{\varepsilon, u}\right\|_{W^{1-1 / p, p}(\Gamma)} \leq C\left\|\partial_{y} L\left(\cdot, y_{\varepsilon, u}(\cdot)\right)\right\|_{L^{p}(\Omega)} .
$$

Inequality (5.3) follows from this estimate and the continuous imbedding $W^{1-1 / p, p}(\Gamma) \subset H^{1-1 / p}(\Gamma)$.

The difference $\varphi_{\varepsilon, u}-\psi_{\varepsilon, u}$ satisfies

$$
\begin{gathered}
-\Delta\left(\varphi_{\varepsilon, u}-\psi_{\varepsilon, u}\right)+\partial_{y} a\left(x, y_{\varepsilon, u}\right)\left(\varphi_{\varepsilon, u}-\psi_{\varepsilon, u}\right)=0 \text { in } \Omega, \\
\varepsilon \partial_{\nu}\left(\varphi_{\varepsilon, u}-\psi_{\varepsilon, u}\right)+\left(\varphi_{\varepsilon, u}-\psi_{\varepsilon, u}\right)=-\varepsilon \partial_{\nu} \psi_{\varepsilon, u} \text { on } \Gamma .
\end{gathered}
$$

Remark 2.3, Theorem 2.2 with $s=t=1-1 / p$ and inequality (5.2) lead to

$$
\left\|\varphi_{\varepsilon, u}-\psi_{\varepsilon, u}\right\|_{H^{1-1 / p}(\Gamma)} \leq C \varepsilon\left\|\partial_{\nu} \psi_{\varepsilon, u}\right\|_{H^{1-1 / p}(\Gamma)} \leq C \varepsilon
$$

With the boundary condition of the adjoint equation for the penalized problem (4.2), and taking into account that $\psi_{\varepsilon, u}=0$ on $\Gamma$, we have

$$
\partial_{\nu} \varphi_{\varepsilon, u}=\frac{\varphi_{\varepsilon, u}}{\varepsilon}=\frac{\varphi_{\varepsilon, u}-\psi_{\varepsilon, u}}{\varepsilon}
$$

With this equality and estimate (5.6) we obtain (5.4). 
The next result will be the key point to obtain the error estimate for the control.

Proposition 5.2. Let $u$ belong to $U_{a d} \cap H^{1 / 2}(\Gamma)$. Let $\varphi_{u}$ and $\varphi_{\varepsilon, u}$ be respectively the adjoint and approximate adjoint states corresponding to $u$. Then

$$
\left\|\partial_{\nu} \varphi_{\varepsilon, u}-\partial_{\nu} \varphi_{u}\right\|_{L^{2}(\Gamma)} \leq C \varepsilon^{1-1 / p}
$$

where $C$ is a constant that depends on $R$, an upper bound for $\|u\|_{L^{\infty}(\Omega)}+\|u\|_{H^{1 / 2}(\Omega)},\|a(\cdot, 0)\|_{L^{p}(\Omega)},\left\|\Psi_{L, K}\right\|_{L^{p}(\Omega)}$, $C_{a, K}$ and $C_{L, K}$, and $K$ is the constant given in Remark 3.3.

Proof. Let us take $\psi_{\varepsilon, u}$ the solution of (5.1). First, from the normal trace result proved in Lemma A.1, we have:

$$
\left\|\partial_{\nu}\left(\varphi_{\varepsilon, u}-\psi_{\varepsilon, u}\right)\right\|_{L^{2}(\Gamma)} \leq C\left(\left\|\varphi_{\varepsilon, u}-\psi_{\varepsilon, u}\right\|_{H^{3 / 2}(\Omega)}+\left\|\Delta\left(\varphi_{\varepsilon, u}-\psi_{\varepsilon, u}\right)\right\|_{L^{2}(\Omega)}\right)
$$

Remark 2.3, Theorem 2.2 for $s=1-1 / p, t=1$, and inequality (5.3) lead to

$$
\left\|\varphi_{\varepsilon, u}-\psi_{\varepsilon, u}\right\|_{H^{3 / 2}(\Omega)} \leq C \varepsilon^{1-1 / p}\left\|\partial_{\nu} \psi_{\varepsilon, u}\right\|_{H^{1-1 / p}(\Gamma)} \leq C \varepsilon^{1-1 / p}
$$

Equation (5.5), assumption (A1) and Theorem 2.2 for $s=t=-1 / 2$ give

$$
\left\|\Delta\left(\varphi_{\varepsilon, u}-\psi_{\varepsilon, u}\right)\right\|_{L^{2}(\Omega)} \leq C\left\|\varphi_{\varepsilon, u}-\psi_{\varepsilon, u}\right\|_{L^{2}(\Omega)} \leq C \varepsilon\left\|\partial_{\nu} \psi_{\varepsilon, u}\right\|_{H^{-1 / 2}(\Gamma)} \leq C \varepsilon
$$

Collecting together estimates (5.7)-(5.9), we obtain

$$
\left\|\partial_{\nu}\left(\varphi_{\varepsilon, u}-\psi_{\varepsilon, u}\right)\right\|_{L^{2}(\Gamma)} \leq C \varepsilon^{1-1 / p} .
$$

On the other hand, the difference $\varphi_{u}-\psi_{\varepsilon, u}$ satisfies the equation

$$
\begin{aligned}
-\Delta\left(\varphi_{u}-\psi_{\varepsilon, u}\right)+\partial_{y} a\left(x, y_{u}\right)\left(\varphi_{u}-\psi_{\varepsilon, u}\right) & =\left(\partial_{y} a\left(x, y_{\varepsilon, u}\right)-\partial_{y} a\left(x, y_{u}\right)\right) \psi_{\varepsilon, u}+\partial_{y} L\left(x, y_{u}\right)-\partial_{y} L\left(x, y_{\varepsilon, u}\right) \text { in } \Omega \\
\varphi_{u}-\psi_{\varepsilon, u} & =0 \text { on } \Gamma
\end{aligned}
$$

We want to estimate the normal derivative of the solution. We proceed as follows. Assumptions (A1) and (A2) tell us that the second derivatives with respect to $y$ of $a(\cdot, y)$ and $L(\cdot, y)$ are bounded, and hence their first derivatives are Lipschitz. Thus we have

$$
\left|\partial_{y} a\left(x, y_{\varepsilon, u}(x)\right)-\partial_{y} a\left(x, y_{u}(x)\right)\right| \leq C_{a, K}\left|y_{u}(x)-y_{\varepsilon, u}(x)\right|
$$

and

$$
\left|\partial_{y} L\left(x, y_{u}(x)\right)-\partial_{y} L\left(x, y_{\varepsilon, u}(x)\right)\right| \leq C_{L, K}\left|y_{u}(x)-y_{\varepsilon, u}(x)\right|
$$

With these inequalities and (5.2), we deduce that the norm in $L^{2}(\Omega)$ of the second member of equation (5.11) is bounded by $C\left\|y_{u}-\bar{y}_{\varepsilon, u}\right\|_{L^{2}(\Omega)}$. Following [11], we can then deduce that

$$
\left\|\varphi_{u}-\psi_{\varepsilon, u}\right\|_{H^{2}(\Omega)} \leq C\left\|y_{u}-y_{\varepsilon, u}\right\|_{L^{2}(\Omega)}
$$

Taking Theorem 3.4 into account, we obtain

$$
\left\|\partial_{\nu}\left(\varphi_{u}-\psi_{\varepsilon, u}\right)\right\|_{L^{2}(\Gamma)} \leq C\left\|\varphi_{u}-\psi_{\varepsilon, u}\right\|_{H^{2}(\Omega)} \leq C\left\|y_{u}-y_{\varepsilon, u}\right\|_{L^{2}(\Omega)} \leq C \varepsilon
$$

The result of the proposition follows from estimates (5.10) and (5.12). 
Proposition 5.3. For $0<\varepsilon<1$, let $\bar{u}_{\varepsilon} \in L^{\infty}(\Gamma)$ be a local solution of $\left(\mathrm{P}_{\varepsilon}\right)$. Then the following holds.

(a) The sequence $\left\{\bar{u}_{\varepsilon}\right\}$ is bounded in $H^{1-1 / p}(\Gamma)$.

(b) There exists at least a subsequence extracted from $\left\{\bar{u}_{\varepsilon}\right\}$ converging to some $\bar{u} \in U_{\text {ad }}$ in $C(\Gamma)$.

(c) Let $\left\{\bar{u}_{\varepsilon}\right\}$ a subsequence converging to some $\bar{u} \in U_{a d}$. If each element $\bar{u}_{\varepsilon}$ is a global solution of $\left(\mathrm{P}_{\varepsilon}\right)$, then $\bar{u} \in U_{a d}$ is a solution of $(\mathrm{P})$.

(d) If the subsequence $\left\{\bar{u}_{\varepsilon}\right\}$ converges to $\bar{u}$ uniformly on $\Gamma$, and if $\bar{u}$ is a local solution of $(\mathrm{P})$, then $\left\{\partial_{\nu} \bar{\varphi}_{\varepsilon}\right\}$ converges uniformly to $\partial_{\nu} \bar{\varphi}$, where $\bar{\varphi}$ and $\bar{\varphi}_{\varepsilon}$ are given in Lemmas 4.1 and 4.2 .

Proof. (a) The first claim follows immediately from (5.4) and (4.3). Since $p>2,2(1-1 / p)>1$ and $H^{1-1 / p}(\Gamma)$ is continuously imbedded in $C(\Gamma)$. Thus $\left\{\bar{u}_{\varepsilon}\right\}$ is included in $C(\Gamma)$.

(b) Since $2(1-1 / p)>1, H^{1-1 / p}(\Gamma)$ is compactly imbedded in $C(\Gamma)$, and hence there is a subsequence of $\bar{u}_{\varepsilon}$ converging uniformly on $\Gamma$ to some $\bar{u} \in C(\Gamma) \cap U_{a d}$.

(c) Since $\left\{\bar{u}_{\varepsilon}\right\}$ is uniformly bounded in $C(\Gamma)$ and in $H^{1 / 2}(\Gamma)$, we can use Theorem 3.4 to deduce

$$
\left\|y_{\bar{u}_{\varepsilon}}-\bar{y}_{\varepsilon}\right\|_{L^{2}(\Omega)} \leq C \varepsilon,
$$

where $y_{\varepsilon}=y_{\varepsilon, \bar{u}_{\varepsilon}}$. Using Lemma 3.1, we have

$$
\left\|y_{\bar{u}}-\bar{y}_{\varepsilon}\right\|_{L^{2}(\Omega)} \leq\left\|y_{\bar{u}}-y_{\bar{u}_{\varepsilon}}\right\|_{L^{2}(\Omega)}+\left\|y_{\bar{u}_{\varepsilon}}-\bar{y}_{\varepsilon}\right\|_{L^{2}(\Omega)} \leq C\left(\left\|\bar{u}-\bar{u}_{\varepsilon}\right\|_{L^{2}(\Omega)}+\varepsilon\right) .
$$

So we have $\bar{y}_{\varepsilon} \rightarrow y_{\bar{u}}$ in $L^{2}(\Omega)$. Let $\hat{u}$ be a solution of (P). Since $\bar{u}$ belongs to $U_{a d}$ and $\left\{\bar{y}_{\varepsilon}\right\}$ is uniformly bounded in $L^{\infty}(\Omega)$, we can write

$$
J(\bar{u}) \leq \liminf J_{\varepsilon}\left(\bar{u}_{\varepsilon}\right) \leq \limsup J_{\varepsilon}\left(\bar{u}_{\varepsilon}\right) \leq \lim \sup J_{\varepsilon}(\hat{u})=J(\hat{u}),
$$

and thus $\bar{u}$ is also a solution of $(\mathrm{P})$.

(d) We now check the convergence of $\left\{\partial_{\nu} \bar{\varphi}_{\varepsilon}\right\}$ in $L^{\infty}(\Gamma)$.

Let us introduce $\varphi_{\bar{u}_{\varepsilon}}$ the exact adjoint state associated with the approximate solution $y_{\bar{u}_{\varepsilon}}$. Applying the triangle inequality we have

$$
\left\|\partial_{\nu} \bar{\varphi}_{\varepsilon}-\partial_{\nu} \bar{\varphi}\right\|_{L^{2}(\Gamma)} \leq\left\|\partial_{\nu} \bar{\varphi}_{\varepsilon}-\partial_{\nu} \varphi_{\bar{u}_{\varepsilon}}\right\|_{L^{2}(\Gamma)}+\left\|\partial_{\nu} \varphi_{\bar{u}_{\varepsilon}}-\partial_{\nu} \bar{\varphi}\right\|_{L^{2}(\Gamma)} .
$$

Since $\left\{\bar{u}_{\varepsilon}\right\}$ is bounded in $H^{1 / 2}(\Gamma)$, we can apply Proposition 5.2 and we obtain

$$
\left\|\partial_{\nu} \bar{\varphi}_{\varepsilon}-\partial_{\nu} \varphi_{\bar{u}_{\varepsilon}}\right\|_{L^{2}(\Gamma)} \leq C \varepsilon^{1-1 / p} .
$$

To show that the second term also converges to zero, let us write the equation satisfied by the difference $\varphi_{\bar{u}_{\varepsilon}}-\bar{\varphi}$ :

$$
\begin{aligned}
-\Delta\left(\varphi_{\bar{u}_{\varepsilon}}-\bar{\varphi}\right)+\partial_{y} a(x, \bar{y})\left(\varphi_{\bar{u}_{\varepsilon}}-\bar{\varphi}\right) & =\left(\partial_{y} a(x, \bar{y})-\partial_{y} a\left(x, y_{\bar{u}_{\varepsilon}}\right)\right) \varphi_{\bar{u}_{\varepsilon}}+\partial_{y} L\left(x, y_{\bar{u}_{\varepsilon}}\right)-\partial_{y} L(x, \bar{y}) \text { in } \Omega \\
\varphi_{\bar{u}_{\varepsilon}}-\bar{\varphi} & =0 \text { on } \Gamma .
\end{aligned}
$$

As we did for equation (5.11), and taking into account that $\varphi_{\bar{u}_{\varepsilon}}$ is uniformly bounded in $L^{\infty}(\Omega)$ (cf. [17]), we deduce that the norm in $L^{2}(\Omega)$ of the second member of equation is bounded by $C\left\|\bar{y}-\bar{y}_{\bar{u}_{\varepsilon}}\right\|_{L^{2}(\Omega)}$. Following $[11]$ and using Lemma 3.1, we can then deduce that

$$
\left\|\partial_{\nu} \varphi_{\bar{u}_{\varepsilon}}-\partial_{\nu} \bar{\varphi}\right\|_{L^{2}(\Gamma)} \leq C\left\|\varphi_{\bar{u}_{\varepsilon}}-\bar{\varphi}\right\|_{H^{2}(\Omega)} \leq C\left\|\bar{y}-\bar{y}_{\bar{u}_{\varepsilon}}\right\|_{L^{2}(\Omega)} \leq C\left\|\bar{u}-\bar{u}_{\varepsilon}\right\|_{L^{2}(\Gamma)} .
$$

Collecting together the previous estimates we have

$$
\left\|\partial_{\nu} \bar{\varphi}_{\varepsilon}-\partial_{\nu} \bar{\varphi}\right\|_{L^{2}(\Gamma)} \leq C\left(\varepsilon^{1-1 / p}+\left\|\bar{u}-\bar{u}_{\varepsilon}\right\|_{L^{2}(\Gamma)}\right) .
$$


Since $\left\{\bar{u}_{\varepsilon}\right\}$ converges to $\bar{u}$ uniformly on $\Gamma$, we first deduce that $\left\{\partial_{\nu} \bar{\varphi}_{\varepsilon}\right\}$ converges to $\partial_{\nu} \bar{\varphi}$ in $L^{2}(\Gamma)$. Knowing that $\left\{\partial_{\nu} \bar{\varphi}_{\varepsilon}\right\}$ is bounded in $H^{1-1 / p, p}(\Gamma)(c f .(5.4))$, it is easy to show that it also converges to $\partial_{\nu} \bar{\varphi}$ in $L^{\infty}(\Gamma)$.

\section{SECOnd order CONDitions And ERror ESTimates}

Since we are dealing with a nonconvex problem, second order sufficient conditions are required to obtain error estimates. Second order conditions have to be written for directions $v \in T_{U_{a d}}(\bar{u})$ such that $J^{\prime}(\bar{u}) v=0$, where $T_{U_{a d}}(\bar{u})$ is the tangent cone at $\bar{u}$ to $U_{a d}$. To characterize these directions, we introduce $\bar{d}(x)=N \bar{u}(x)-\partial_{\nu} \bar{\varphi}(x)$, $\tau \geq 0$ and the following conditions:

$$
\begin{aligned}
& v(x)=0 \text { if }|\bar{d}(x)|>\tau, \\
& v(x) \geq 0 \text { if } \bar{u}(x)=\alpha, \\
& v(x) \leq 0 \text { if } \bar{u}(x)=\beta .
\end{aligned}
$$

For $\tau \geq 0$, we define the cone

For $\rho>0$, we set

$$
C_{\bar{u}}^{\tau}=\left\{v \in L^{2}(\Gamma) \mid v \text { satisfies (6.1)-(6.3) }\right\} .
$$

$$
\begin{aligned}
& B_{\rho}(\bar{u})=\left\{u \in L^{\infty}(\Gamma):\|u-\bar{u}\|_{L^{\infty}(\Gamma)}<\rho\right\}, \\
& \bar{B}_{\rho}(\bar{u})=\left\{u \in L^{\infty}(\Gamma):\|u-\bar{u}\|_{L^{\infty}(\Gamma)} \leq \rho\right\} .
\end{aligned}
$$

We have the following result about second order necessary and sufficient conditions:

Lemma $6.1([5])$. Let $\bar{u} \in U_{a d}$ be a solution of $(\mathrm{P})$. Then

$$
J^{\prime \prime}(\bar{u}) v^{2} \geq 0 \text { for all } v \in C_{\bar{u}}^{0} .
$$

Conversely, let $\bar{u} \in U_{\text {ad }}$ satisfy the first order necessary condition (4.1), and suppose in addition that $\bar{u}$ obeys

$$
J^{\prime \prime}(\bar{u}) v^{2}>0 \text { for all } v \in C_{\bar{u}}^{0} \backslash\{0\} .
$$

Then there exist $\rho>0$ and $\kappa>0$ such that, for all $u \in \bar{B}_{\rho}(\bar{u})$,

$$
J(\bar{u})+\kappa\|\bar{u}-u\|_{L^{2}(\Omega)}^{2} \leq J(u) .
$$

Moreover, there exist $\delta>0$ and $\tau>0$ such that

$$
J^{\prime \prime}(\bar{u}) v^{2} \geq \delta\|v\|_{L^{2}(\Gamma)}^{2} \text { for all } v \in C_{\bar{u}}^{\tau} .
$$

Proposition 6.2. Let $\bar{u} \in U_{\text {ad }}$ be a solution of $(\mathrm{P})$ that satisfies the second order sufficient conditions (6.4). Then there exists $\bar{\varepsilon}>0$ and $\rho>0$ such that for all $0<\varepsilon<\bar{\varepsilon}$, $\left(\mathrm{P}_{\varepsilon}\right)$ has at least one local solution $\bar{u}_{\varepsilon}$ in $B_{\rho}(\bar{u})$. Moreover, the whole sequence $\left\{\bar{u}_{\varepsilon}\right\}$ converges to $\bar{u}$ uniformly on $\Gamma$.

Proof. Consider the problems

and

$$
\left(\mathrm{P}^{\rho}\right)\left\{\begin{array}{l}
\min J(u) \\
u \in U_{a d} \cap \bar{B}_{\rho}(\bar{u}),
\end{array}\right.
$$

$$
\left(\mathrm{P}_{\varepsilon}^{\rho}\right)\left\{\begin{array}{l}
\min J_{\varepsilon}(u) \\
u \in U_{a d} \cap \bar{B}_{\rho}(\bar{u}) .
\end{array}\right.
$$

The set $U_{a d} \cap \bar{B}_{\rho}(\bar{u})$ is closed, convex, bounded, and nonempty because $\bar{u} \in U_{a d} \cap \bar{B}_{\rho}(\bar{u})$. The functional $J_{\varepsilon}$ is lower semicontinuous for the weak topology of $L^{2}(\Gamma)$. Therefore $\left(\mathrm{P}_{\varepsilon}^{\rho}\right)$ has at least one solution $u_{\varepsilon}^{\rho}$. We can apply Proposition 5.3 to these problems, and we obtain that there is a subsequence $\left\{u_{\varepsilon}^{\rho}\right\}$ converging uniformly 
to a solution of $\left(\mathrm{P}^{\rho}\right)$. Due to (6.5), $\bar{u}$ is the unique solution of $\left(\mathrm{P}^{\rho}\right)$. Therefore $u_{\varepsilon}^{\rho} \rightarrow \bar{u}$. Moreover, since every convergent subsequence, converges to the same limit, the whole sequence converges to the same limit $\bar{u}$.

Finally, since the convergence is uniform, there exists some $\bar{\varepsilon}>0$ such that, for all $0<\varepsilon<\bar{\varepsilon}, u_{\varepsilon}^{\rho} \in B_{\rho}(\bar{u})$, and thus $u_{\varepsilon}^{\rho}$ is a local solution of $\left(\mathrm{P}_{\varepsilon}\right)$.

Next we find a bound for the $L^{2}(\Gamma)$ norm of the error in terms of the derivative of $J$.

Lemma 6.3. Suppose that $\bar{u}$ is a local solution of $(\mathrm{P})$ satisfying (6.4), and let $\bar{u}_{\varepsilon}$ be a local solution to $\left(\mathrm{P}_{\varepsilon}\right)$ in $U_{\text {ad }} \cap B_{\rho}(\bar{u})$, where $0<\varepsilon<\bar{\varepsilon}(\bar{\varepsilon}$ and $\rho$ being the parameter and the radius introduced in Prop. 6.2). Then there exists $0<\varepsilon^{*} \leq \bar{\varepsilon}$ such that

$$
\frac{\delta}{2}\left\|\bar{u}-\bar{u}_{\varepsilon}\right\|_{L^{2}(\Gamma)}^{2} \leq\left(J^{\prime}(\bar{u})-J^{\prime}\left(\bar{u}_{\varepsilon}\right)\right)\left(\bar{u}-\bar{u}_{\varepsilon}\right)
$$

for all $0<\varepsilon<\varepsilon^{*}$, where $\delta$ is given in Lemma 6.1 .

Proof. We have

$$
\left(J^{\prime}(\bar{u})-J^{\prime}\left(\bar{u}_{\varepsilon}\right)\right)\left(\bar{u}-\bar{u}_{\varepsilon}\right)=J^{\prime \prime}(\bar{u})\left(\bar{u}-\bar{u}_{\varepsilon}\right)^{2}+\left(J^{\prime \prime}\left(u_{\theta}\right)-J^{\prime \prime}(\bar{u})\right)\left(\bar{u}-\bar{u}_{\varepsilon}\right)^{2},
$$

for some $\theta \in(0,1)$ and $u_{\theta}=\bar{u}+\theta\left(\bar{u}_{\varepsilon}-\bar{u}\right)$.

From Proposition 6.2, we know that $\left\{\bar{u}_{\varepsilon}\right\}$ converges to $\bar{u}$ uniformly on $\Gamma$. Therefore we can make use of Proposition 5.3. Let us denote by $\bar{d}_{\varepsilon}(x)=N \bar{u}_{\varepsilon}(x)-\partial_{\nu} \bar{\varphi}_{\varepsilon}(x)$ the derivative $J_{\varepsilon}^{\prime}\left(\bar{u}_{\varepsilon}\right)$. From Proposition 5.3, we know that $\bar{d}_{\varepsilon} \rightarrow \bar{d}$ uniformly on $\Gamma$. Let us check that $v=\bar{u}_{\varepsilon}-\bar{u}$ belongs to $C_{\bar{u}}^{\tau}$, where $\tau$ is the parameter introduced in Lemma 6.1. Since $\bar{u}_{\varepsilon} \in U_{a d}$, it is obvious that $v$ satisfies the sign conditions (6.2) and (6.3). If $\bar{d}(x)>\tau$, using (4.1) we have $\bar{u}(x)=\alpha$. Moreover, due to the uniform convergence $\bar{d}_{\varepsilon} \rightarrow \bar{d}$, there exists $\varepsilon_{+}>0$ such that, for all $0<\varepsilon<\varepsilon_{+}, \bar{d}_{\varepsilon}(x)>\tau / 2$. Using (4.3) we obtain $\bar{u}_{\varepsilon}(x)=\alpha$. And hence $v(x)=0$. With a similar argument we obtain that if $\bar{d}(x)<-\tau$ then there exists $\varepsilon_{-}>0$ such that, for all $0<\varepsilon<\varepsilon_{-}, v(x)=0$. Therefore, for all $0<\varepsilon<\varepsilon_{1}=\min \left\{\varepsilon_{+}, \varepsilon_{-}\right\}, \bar{u}_{\varepsilon}-\bar{u}$ belongs to $C_{\bar{u}}^{\tau}$.

From (6.5) we deduce

$$
\delta\left\|\bar{u}-\bar{u}_{\varepsilon}\right\|_{L^{2}(\Gamma)}^{2} \leq J^{\prime \prime}(\bar{u})\left(\bar{u}-\bar{u}_{\varepsilon}\right)^{2} .
$$

Let us now show that $\left(J^{\prime \prime}\left(u_{\theta}\right)-J^{\prime \prime}(\bar{u})\right)\left(\bar{u}-\bar{u}_{\varepsilon}\right)^{2}$ is small. Let us define $\bar{\xi}$ and $\xi_{\theta}$ as the solutions of the linearized state equations around $\bar{u}$ and $u_{\theta}$ in the direction of $v=\bar{u}-\bar{u}_{\varepsilon}$ :

$$
\begin{gathered}
-\Delta \bar{\xi}+\partial_{y} a(x, \bar{y}) \bar{\xi}=0 \text { in } \Omega, \bar{\xi}=\bar{u}-\bar{u}_{\varepsilon} \text { on } \Gamma, \\
-\Delta \xi_{\theta}+\partial_{y} a\left(x, y_{\theta}\right) \xi_{\theta}=0 \text { in } \Omega, \xi_{\theta}=\bar{u}-\bar{u}_{\varepsilon} \text { on } \Gamma,
\end{gathered}
$$

where $y_{\theta}=y_{u_{\theta}}$. Let us denote by $\varphi_{\theta}=\varphi_{u_{\theta}}$ the adjoint state associated with $u_{\theta}=\bar{u}+\theta\left(\bar{u}_{\varepsilon}-\bar{u}\right)$. Then

$$
J^{\prime \prime}(\bar{u})\left(\bar{u}-\bar{u}_{\varepsilon}\right)^{2}=\int_{\Omega}\left(\partial_{y y}^{2} L(x, \bar{y})-\partial_{y y}^{2} a(x, \bar{y}) \bar{\varphi}\right) \bar{\xi}^{2} \mathrm{~d} x+N \int_{\Gamma}\left(\bar{u}-\bar{u}_{\varepsilon}\right)^{2} \mathrm{~d} \sigma(x),
$$

and

$$
J^{\prime \prime}\left(u_{\theta}\right)\left(\bar{u}-\bar{u}_{\varepsilon}\right)^{2}=\int_{\Omega}\left(\partial_{y y}^{2} L\left(x, y_{\theta}\right)-\partial_{y y}^{2} a\left(x, y_{\theta}\right) \varphi_{\theta}\right) \xi_{\theta}^{2} \mathrm{~d} x+N \int_{\Gamma}\left(\bar{u}-\bar{u}_{\varepsilon}\right)^{2} \mathrm{~d} \sigma(x) .
$$

Since $y_{\theta}$ uniformly convergences to $\bar{y}$ as $\varepsilon \rightarrow 0$, there exists $\varepsilon_{2}>0$ such that

$$
\left|\left(J^{\prime \prime}\left(u_{\theta}\right)-J^{\prime \prime}(\bar{u})\right)\left(\bar{u}-\bar{u}_{\varepsilon}\right)^{2}\right| \leq \frac{\delta}{2}\left\|\bar{u}-\bar{u}_{\varepsilon}\right\|_{L^{2}(\Gamma)}^{2}
$$


for all $0<\varepsilon<\varepsilon_{2}$. Let us see this with some more detail. With assumption (A2), the uniform boundness of $y_{\theta}$ and $\varphi_{\theta}$ and assumption (A1) we get

$$
\begin{aligned}
& \left|\left(J^{\prime \prime}\left(u_{\theta}\right)-J^{\prime \prime}(\bar{u})\right)\left(\bar{u}-\bar{u}_{\varepsilon}\right)^{2}\right|=\left|\int_{\Omega}\left(\partial_{y y}^{2} L(x, \bar{y})-\partial_{y y}^{2} a(x, \bar{y}) \bar{\varphi}\right) \bar{\xi}^{2} \mathrm{~d} x-\int_{\Omega}\left(\partial_{y y}^{2} L\left(x, y_{\theta}\right)-\partial_{y y}^{2} a\left(x, y_{\theta}\right) \varphi_{\theta}\right) \xi_{\theta}^{2} \mathrm{~d} x\right| \\
& \leq \int_{\Omega}\left(\left|\partial_{y y}^{2} L(x, \bar{y})-\partial_{y y}^{2} L\left(x, y_{\theta}\right)\right|+\left|\partial_{y y}^{2} a(x, \bar{y}) \bar{\varphi}-\partial_{y y}^{2} a\left(x, y_{\theta}\right) \varphi_{\theta}\right|\right) \bar{\xi}^{2} \mathrm{~d} x \\
& \quad+\int_{\Omega}\left|\partial_{y y}^{2} L\left(x, y_{\theta}\right)-\partial_{y y}^{2} a\left(x, y_{\theta}\right) \varphi_{\theta}\right|\left|\bar{\xi}^{2}-\xi_{\theta}^{2}\right| \mathrm{d} x \\
& \leq C_{L, K} \int_{\Omega}\left|\bar{y}-y_{\theta}\right| \bar{\xi}^{2} \mathrm{~d} x+\int_{\Omega}\left|\partial_{y y}^{2} a(x, \bar{y})-\partial_{y y}^{2} a\left(x, y_{\theta}\right)\right||\bar{\varphi}| \bar{\xi}^{2} \mathrm{~d} x \\
& \quad+\int_{\Omega}\left|\partial_{y y}^{2} a\left(x, y_{\theta}\right)\right|\left|\bar{\varphi}-\varphi_{\theta}\right| \bar{\xi}^{2} \mathrm{~d} x+\left(C_{L, K}+K C_{a, K}\right) \int_{\Omega}\left|\bar{\xi}-\xi_{\theta}\right|\left|\bar{\xi}+\xi_{\theta}\right| \mathrm{d} x \\
& \leq C_{L, K}\left\|\bar{y}-y_{\theta}\right\|_{L^{\infty}(\Omega)}|| \bar{\xi} \|_{L^{2}(\Omega)}+K C_{a, K} \int_{\Omega}\left|\bar{y}-y_{\theta}\right| \bar{\xi}^{2} \mathrm{~d} x+C_{a, K} \int_{\Omega}\left|\bar{\varphi}-\varphi_{\theta}\right| \bar{\xi}^{2} \mathrm{~d} x \\
& \quad+\left(C_{L, K}+K C_{a, K}\right)\left\|\bar{\xi}-\xi_{\theta}\right\|_{L^{2}(\Omega)}\left\|\bar{\xi}+\xi_{\theta}\right\|_{L^{2}(\Omega)},
\end{aligned}
$$

where $K>0$ is given in Remark 3.3. From the equation satisfied by $\bar{\xi}$, we know that there exists a constant $C(K)$ such that $\|\bar{\xi}\|_{L^{2}(\Omega)} \leq C(K)\left\|\bar{u}-\bar{u}_{\varepsilon}\right\|_{L^{2}(\Gamma)}$. The uniform convergence of $\left(\bar{u}_{\varepsilon}\right)_{\varepsilon}$ to $\bar{u}$ implies the uniform convergence in $\Omega$ of $y_{\theta}$ to $\bar{y}$ and of $\varphi_{\theta}$ to $\bar{\varphi}$ (see Lem. 3.1). We can deduce that there exists $\varepsilon_{3}>0$ such that for all $0<\varepsilon<\varepsilon_{3}$

$$
C_{L, K}\left\|\bar{y}-y_{\theta}\right\|_{L^{\infty}(\Omega)}\|\bar{\xi}\|_{L^{2}(\Omega)}+K C_{a, K} \int_{\Omega}\left|\bar{y}-y_{\theta}\right| \bar{\xi}^{2} \mathrm{~d} x+C_{a, K} \int_{\Omega}\left|\bar{\varphi}-\varphi_{\theta}\right| \bar{\xi}^{2} \mathrm{~d} x \leq \frac{\delta}{4}\left\|\bar{u}-\bar{u}_{\varepsilon}\right\|_{L^{2}(\Gamma)}^{2} .
$$

From the equations satisfied by $\bar{\xi}$ and $\xi_{\theta}$, we have

$$
\begin{aligned}
& -\Delta\left(\bar{\xi}-\xi_{\theta}\right)+\partial_{y} a(x, \bar{y})\left(\bar{\xi}-\xi_{\theta}\right)=\left(\partial_{y} a\left(x, y_{\theta}\right)-\partial_{y} a(x, \bar{y})\right) \xi_{\theta} \text { in } \Omega, \\
& \bar{\xi}-\xi_{\theta}=0 \text { on } \Gamma
\end{aligned}
$$

and

$$
\begin{aligned}
& -\Delta\left(\bar{\xi}+\xi_{\theta}\right)+\partial_{y} a(x, \bar{y})\left(\bar{\xi}+\xi_{\theta}\right)=\left(\partial_{y} a(x, \bar{y})-\partial_{y} a\left(x, y_{\theta}\right)\right) \xi_{\theta} \text { in } \Omega, \\
& \bar{\xi}+\xi_{\theta}=2\left(\bar{u}-\bar{u}_{\varepsilon}\right) \quad \text { on } \Gamma .
\end{aligned}
$$

Using assumption (A1), we have

$$
\begin{gathered}
\left\|\bar{\xi}-\xi_{\theta}\right\|_{L^{2}(\Omega)} \leq C(K)\left\|\bar{y}-y_{\theta}\right\|_{L^{\infty}(\Omega)}\left\|\xi_{\theta}\right\|_{L^{2}(\Omega)}, \\
\left\|\bar{\xi}+\xi_{\theta}\right\|_{L^{2}(\Omega)} \leq C(K)\left(\left\|\bar{y}-y_{\theta}\right\|_{L^{\infty}(\Omega)}\left\|\xi_{\theta}\right\|_{L^{2}(\Omega)}+\left\|\bar{u}-\bar{u}_{\varepsilon}\right\|_{L^{2}(\Gamma)}\right) .
\end{gathered}
$$

From the equation satisfied by $\xi_{\theta}$, we know that there exists a constant $C(K)$ such that $\left\|\xi_{\theta}\right\|_{L^{2}(\Omega)} \leq C(K) \| \bar{u}-$ $\bar{u}_{\varepsilon} \|_{L^{2}(\Gamma)}$. Using again the uniform convergence of the states, we deduce that there exists $\varepsilon_{4}>0$ such that

$$
\left(C_{L, K}+K C_{a, K}\right)\left\|\bar{\xi}-\xi_{\theta}\right\|_{L^{2}(\Omega)}\left\|\bar{\xi}+\xi_{\theta}\right\|_{L^{2}(\Omega)} \leq \frac{\delta}{4}\left\|\bar{u}-\bar{u}_{\varepsilon}\right\|_{L^{2}(\Gamma)}^{2},
$$

for all $0<\varepsilon<\varepsilon_{4}$. Therefore inequality (6.6) follows from inequalities (6.7) and (6.8) for $\varepsilon_{2}=\min \left\{\varepsilon_{3}, \varepsilon_{4}\right\}$.

The proof is complete by taking $\varepsilon^{*}=\min \left\{\varepsilon_{1}, \varepsilon_{2}\right\}$.

Finally, we are able to prove the main result of the paper. 
Proof of Theorem 2.1. Since $\bar{u}_{\varepsilon} \in U_{a d}$, from first order optimality conditions for (P) we deduce $J^{\prime}(\bar{u})\left(\bar{u}-\bar{u}_{\varepsilon}\right) \leq 0$. Similarly, since $\bar{u} \in U_{a d}$, we have $J_{\varepsilon}^{\prime}\left(\bar{u}_{\varepsilon}\right)\left(\bar{u}-\bar{u}_{\varepsilon}\right) \geq 0$. With the previous lemma we can write

$$
\begin{aligned}
& \frac{\delta}{2}\left\|\bar{u}-\bar{u}_{\varepsilon}\right\|_{L^{2}(\Gamma)}^{2} \leq\left(J^{\prime}(\bar{u})-J^{\prime}\left(\bar{u}_{\varepsilon}\right)\right)\left(\bar{u}-\bar{u}_{\varepsilon}\right) \leq\left(J_{\varepsilon}^{\prime}\left(\bar{u}_{\varepsilon}\right)-J^{\prime}\left(\bar{u}_{\varepsilon}\right)\right)\left(\bar{u}-\bar{u}_{\varepsilon}\right) \\
& =\int_{\Gamma}\left(\partial_{\nu} \bar{\varphi}_{\varepsilon}-\partial_{\nu} \varphi_{\bar{u}_{\varepsilon}}\right)\left(\bar{u}-\bar{u}_{\varepsilon}\right) \mathrm{d} \sigma(x) \leq\left\|\partial_{\nu} \bar{\varphi}_{\varepsilon}-\partial_{\nu} \varphi_{\bar{u}_{\varepsilon}}\right\|_{L^{2}(\Gamma)}\left\|\bar{u}-\bar{u}_{\varepsilon}\right\|_{L^{2}(\Gamma)} .
\end{aligned}
$$

Since $\bar{u}_{\varepsilon}$ is uniformly bounded in $H^{1 / 2}(\Gamma)$, we can use Proposition 5.2 to complete the proof.

\section{Linear quadratic PRoblems and unbounded CONTROLS}

In [2] the authors consider a linear quadratic control problem with controls which are not bounded in $L^{\infty}(\Gamma)$ (in that case we shall speak of unbounded controls. It corresponds to the case when $\alpha=-\infty$ and $\beta=\infty$ ). They establish an estimate of order $\sqrt{\varepsilon}$ for the error in $L^{2}(\Gamma)$ (cf. equation (29) in [2]). That estimate can be improved with our method.

Theorem 7.1. Let $L(x, y)=\frac{1}{2}\left(y-y_{d}(x)\right)^{2}$, where $y_{d} \in L^{\bar{p}}(\Omega), a(x, y)=0, \alpha=-\infty$, and $\beta=\infty$. Then we have

$$
\left\|\bar{u}-\bar{u}_{\varepsilon}\right\|_{L^{2}(\Omega)} \leq C \varepsilon^{1-1 / p}
$$

for all $0<\varepsilon<1$. (Let us recall that $p=\min \left\{\bar{p}, p_{\Omega}\right\}$ if $\omega>\pi / 2$ and $p=\bar{p}$ if $\omega \leq \pi / 2$ and $\bar{p}<\infty$.)

All the assumptions of our original problem are fulfilled, except that the set of admissible controls is not bounded in $L^{\infty}(\Gamma)$. So, first of all, we will prove that the solutions are uniformly bounded in $L^{\infty}(\Gamma)$.

Lemma 7.2. For every $0<\varepsilon<1$, the penalized problem $\left(\mathrm{P}_{\varepsilon}\right)$ has a unique global solution $\bar{u}_{\varepsilon} \in H^{1-1 / p}(\Gamma)$, and $\left\{\bar{u}_{\varepsilon}\right\}$ is uniformly bounded in $H^{1-1 / p}(\Gamma)$. In particular, this is a bounded family in $C(\Gamma)$ that converges uniformly to $\bar{u}$, the unique solution of $(\mathrm{P})$. Moreover, $\bar{u}$ belongs to $H^{1-1 / p}(\Gamma)$, and hence it is a continuous function.

Proof. Now $(\mathrm{P})$ and $\left(\mathrm{P}_{\varepsilon}\right)$ are linear quadratic problems. Existence and uniqueness of a global solution in $L^{2}(\Gamma)$ is easily deduced by classical methods. The state and adjoint state equations for the penalized problem can be written in the form

$$
\begin{gathered}
\int_{\Omega} \nabla \bar{y}_{\varepsilon} \nabla z \mathrm{~d} x+\frac{1}{\varepsilon} \int_{\Gamma} \bar{y}_{\varepsilon} z \mathrm{~d} \sigma(x)=\frac{1}{\varepsilon} \int_{\Gamma} \bar{u}_{\varepsilon} z \mathrm{~d} \sigma(x) \text { for all } z \in H^{1}(\Omega), \\
\int_{\Omega} \nabla w \nabla \bar{\varphi}_{\varepsilon} \mathrm{d} x+\frac{1}{\varepsilon} \int_{\Gamma} w \bar{\varphi}_{\varepsilon} \mathrm{d} \sigma(x)=\int_{\Omega}\left(\bar{y}_{\varepsilon}-y_{d}\right) w \mathrm{~d} x \text { for all } w \in H^{1}(\Omega) .
\end{gathered}
$$

Taking $z=\bar{\varphi}_{\varepsilon}$ and $w=\bar{y}_{\varepsilon}$, and noting that the left hand sides of both equalities are equal, we obtain from the right hand sides

$$
\frac{1}{\varepsilon} \int_{\Gamma} \bar{u}_{\varepsilon} \bar{\varphi}_{\varepsilon} \mathrm{d} \sigma(x)=\int_{\Omega}\left(\bar{y}_{\varepsilon}-y_{d}\right) \bar{y}_{\varepsilon} \mathrm{d} x
$$

From the first order optimality conditions, we know that $\bar{u}_{\varepsilon}=\frac{1}{N} \partial_{\nu} \bar{\varphi}_{\varepsilon}$ and from the boundary condition of the adjoint state equation, we know that $\bar{\varphi}_{\varepsilon}=-\varepsilon \partial_{\nu} \bar{\varphi}_{\varepsilon}$. Thus, we have

$$
-\frac{1}{N} \int_{\Gamma}\left(\partial_{\nu} \bar{\varphi}_{\varepsilon}\right)^{2} \mathrm{~d} \sigma(x)=\int_{\Omega}\left(\bar{y}_{\varepsilon}^{2}-y_{d} \bar{y}_{\varepsilon}\right) \mathrm{d} x
$$

and

$$
\int_{\Omega} \bar{y}_{\varepsilon}^{2} \mathrm{~d} x+\frac{1}{N} \int_{\Gamma}\left(\partial_{\nu} \bar{\varphi}_{\varepsilon}\right)^{2} \mathrm{~d} \sigma(x)=\int_{\Omega} y_{d} y_{\varepsilon} \mathrm{d} x .
$$


Using the Cauchy-Schwarz inequality in the above identity, we first obtain $\left\|\bar{y}_{\varepsilon}\right\|_{L^{2}(\Omega)} \leq\left\|y_{d}\right\|_{L^{2}(\Omega)}$. Then $\left\|\partial_{\nu} \bar{\varphi}_{\varepsilon}\right\|_{L^{2}(\Gamma)} \leq \sqrt{N}\left\|y_{d}\right\|_{L^{2}(\Omega)}$ and from the first order optimality conditions we conclude that there exists $C>0$ such that

$$
\left\|\bar{u}_{\varepsilon}\right\|_{L^{2}(\Gamma)} \leq C .
$$

From Theorem 2.2 (for $t=s=0$ ), we know that $\left\{\bar{y}_{\varepsilon}\right\}$ is bounded in $H^{1 / 2}(\Omega)$, and by Sobolev imbeddings, it is also bounded in $L^{4}(\Omega)$.

Now, we repeat the arguments of the proof of Lemma 5.1. Let us consider $\bar{\psi}_{\varepsilon}$ the solution of

$$
-\Delta \bar{\psi}_{\varepsilon}=\bar{y}_{\varepsilon}-y_{d} \text { in } \Omega, \quad \bar{\psi}_{\varepsilon}=0 \text { on } \Gamma .
$$

Set $q=\min \{p, 4\}$. We have $q>2$. Using Lemma A.2, estimate (A.3) (or estimate (3.5)), we deduce that

$$
\left\|\partial_{\nu} \bar{\psi}_{\varepsilon}\right\|_{H^{1-1 / q}(\Gamma)} \leq C\left\|\partial_{\nu} \bar{\psi}_{\varepsilon}\right\|_{W^{1-1 / q, q}(\Gamma)} \leq C\left\|\bar{y}_{\varepsilon}-y_{d}\right\|_{L^{q}(\Omega)} \leq C .
$$

The difference $\bar{\varphi}_{\varepsilon}-\bar{\psi}_{\varepsilon}$ satisfies

$$
-\Delta\left(\bar{\varphi}_{\varepsilon}-\bar{\psi}_{\varepsilon}\right)=0 \text { on } \Omega, \quad \varepsilon \partial_{\nu}\left(\bar{\varphi}_{\varepsilon}-\bar{\psi}_{\varepsilon}\right)+\left(\bar{\varphi}_{\varepsilon}-\bar{\psi}_{\varepsilon}\right)=-\varepsilon \partial_{\nu} \bar{\psi}_{\varepsilon} \text { on } \Gamma .
$$

Theorem 2.2 with $s=t=1-1 / q$ and inequality (7.1) lead to

$$
\left\|\bar{\varphi}_{\varepsilon}-\bar{\psi}_{\varepsilon}\right\|_{H^{1-1 / q}(\Gamma)} \leq C \varepsilon\left\|\partial_{\nu} \bar{\psi}_{\varepsilon}\right\|_{H^{1-1 / q}(\Gamma)} \leq C \varepsilon
$$

With the boundary condition of the adjoint equation for the penalized problem (4.2), and with $\bar{\psi}_{\varepsilon}=0$ on $\Gamma$, we obtain

$$
\left\|\partial_{\nu} \bar{\varphi}_{\varepsilon}\right\|_{H^{1-1 / q}(\Gamma)} \leq C .
$$

Observe that $\bar{u}_{\varepsilon}=\frac{1}{N} \partial_{\nu} \bar{\varphi}_{\varepsilon}$ because there are no bound constraints. Since $q>2, H^{1-1 / q}(\Gamma)$ is continuously imbedded in $C(\Gamma)$, and all the assumptions of Proposition 5.3 are satisfied. So the first claim follows from Proposition 5.3(a). From Proposition 5.3(b) and (c) we know that there exist subsequences that converge uniformly to solutions of $(\mathrm{P})$. In the present case the solution of $(\mathrm{P})$ is unique. Therefore all the sequence converges to $\bar{u}$. Finally, since $\left\{\bar{u}_{\varepsilon}\right\}$ is bounded in $H^{1-1 / p, p}(\Gamma)$, there exists a subsequence weakly convergent in that space to $\bar{u} \in H^{1-1 / p, p}(\Gamma)$ because the limit is unique.

Proof of Theorem 7.1. Thanks to the previous lemma, Propositions 5.2 and 5.3 are still valid. Since the problem is quadratic and strictly convex, the second order sufficient condition (6.4) is trivially satisfied, and we can apply Theorem 2.1.

Remark 7.3. If $\bar{p}=\infty$ and the domain is a square, then $p$ can be chosen arbitrarily big, and the result stated in Theorem 7.1 means that the expected order of convergence for the error in the $L^{2}(\Gamma)$ norm of the control will be $\varepsilon^{1-\delta}$ for all $\delta>0$. This behavior was numerically observed in the second example in [2], pp. 847-848 (see also [2], Fig. 5, and the comments at the end of Sect. 5 in [2]). Nevertheless, it remained unexplained in that reference.

\section{An EXAMPLE}

Let $\Omega=(0,1)^{2}$ be the unit square in $\mathbb{R}^{2}$ and let us set

$$
y_{d}(x)=\frac{1}{|x|^{2 / 3}} .
$$




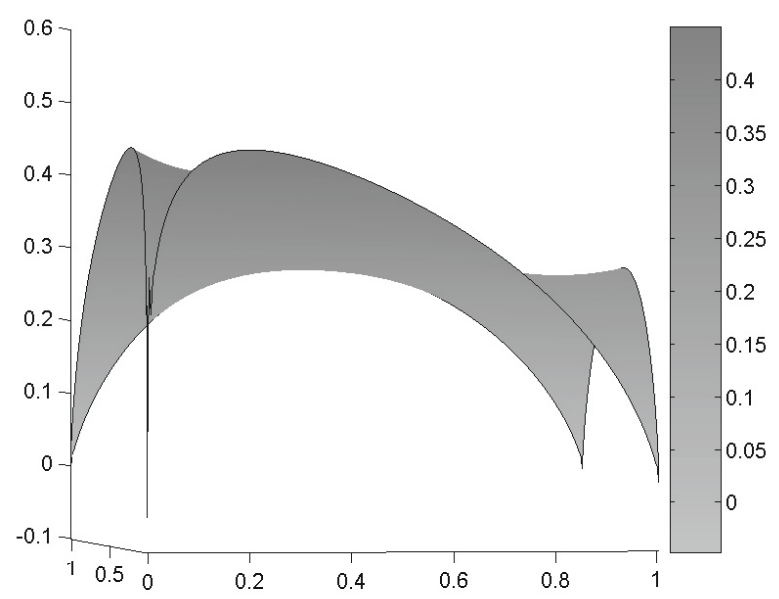

Figure 1. Solution.

We see that $y_{d} \in L^{p}(\Omega)$ for all $p<3$. Now we consider the linear-quadratic control problem

$$
(\mathrm{P})\left\{\begin{array}{l}
\operatorname{Min} J(u)=\frac{1}{2} \int_{\Omega}\left(y_{u}-y_{d}\right)^{2} \mathrm{~d} x+\frac{1}{2} \int_{\Gamma} u^{2} \mathrm{~d} \sigma(x) \\
u \in U_{a d}=\left\{u \in L^{2}(\Gamma):-1 \leq u(x) \leq 1 \text { a.e. } x \in \Gamma\right\} \\
-\Delta y_{u}=0 \text { in } \Omega, \quad y_{u}=u \text { on } \Gamma .
\end{array}\right.
$$

From a strict convexity argument, it follows that this problem has a unique global solution. From Lemma 4.1 we know that there exists $(\bar{y}, \bar{\varphi}, \bar{u})$ belonging to $W^{1, p}(\Omega) \times W^{2, p}(\Omega) \times W^{1-1 / p, p}(\Gamma)$ for all $p<3$, and satisfying the first order optimality conditions.

The penalized problem is

$$
\left(\mathrm{P}_{\varepsilon}\right)\left\{\begin{array}{l}
\operatorname{Min} J_{\varepsilon}(u)=\frac{1}{2} \int_{\Omega}\left(y_{\varepsilon, u}-y_{d}\right)^{2} \mathrm{~d} x+\frac{1}{2} \int_{\Gamma} u^{2} \mathrm{~d} \sigma(x) \\
u \in U_{a d} \\
-\Delta y_{\varepsilon, u}=0 \text { in } \Omega, \quad \varepsilon \partial_{\nu} y_{\varepsilon, u}+y_{\varepsilon, u}=u \text { on } \Gamma .
\end{array}\right.
$$

For every $\varepsilon>0$ we have a unique global solution $\bar{u}_{\varepsilon} \in W^{2-1 / p, p}(\Gamma)$.

Since we do not have the analytic solutions, we are going to solve these problems numerically. For that purpose we approximate the controls by continuous piecewise linear functions and we apply a primal dual active set strategy for the optimization. To solve the PDEs arising in the optimization process we use the finite element method. Of course the Dirichlet conditions are imposed exactly at the boundary nodes, and not treated by penalization. All the software, except for the mesh generator, has been programmed by us with MATLAB. To this aim, we have considered a regular family of triangulations $\left\{\mathcal{T}_{h}\right\}_{h}$, where, as usual, $h$ denotes the mesh size. Let $\left\{x_{j}\right\}_{1 \leq j \leq N(h)}$ be the nodes in the boundary, starting at the origin and counting counterclockwise and $x_{N(h)+1}=x_{1}$. The space of discretized controls is

$$
U^{h}=\left\{u^{h} \in C(\Gamma): u_{\mid\left[x_{j}, x_{j+1}\right]}^{h} \in \mathcal{P}^{1}\right\}
$$


TABLE 1. $\left\|\bar{u}^{h}\right\|_{H^{s}(\Gamma)}$.

\begin{tabular}{|c|c|c|c|c|c|c|}
\hline$n$ & $s=0.5$ & $s=0.6$ & $=0.7$ & $=0.8$ & $s=0.9$ & $s=1$ \\
\hline 3 & 1.8654 & 2.1497 & 2.5093 & 2.9609 & 3.5245 & 2.7290 \\
\hline 4 & 1.7183 & 2.0528 & 2.5065 & 3.1161 & 3.9289 & 3.1445 \\
\hline 5 & 1.6163 & 1.9831 & 2.5168 & 3.2843 & 4.3783 & 3.6347 \\
\hline 6 & 1.5465 & 1.9323 & 2.5333 & 3.4578 & 4.8658 & 4.2037 \\
\hline 7 & 1.4984 & 1.8931 & 2.5486 & 3.6261 & 5.3788 & 4.8479 \\
\hline 8 & 1.4652 & 1.8619 & 2.5603 & 3.7843 & 5.9093 & 5.5659 \\
\hline 9 & 1.4427 & 1.8372 & 2.5685 & 3.9314 & 6.4546 & 6.3608 \\
\hline
\end{tabular}

TABLE 2. $\left\|r_{h}\right\|_{H^{s}(0,1)}$.

\begin{tabular}{|c|c|c|c|c|c|c|}
\hline$n$ & $s=0.5$ & $s=0.6$ & $=0.7$ & $s=0.8$ & $s=0.9$ & $s=1$ \\
\hline 3 & 1.6038 & 1.8276 & 2.1164 & 2.4861 & 2.9559 & 2.3120 \\
\hline 4 & 1.5416 & 1.8143 & 2.1928 & 2.7106 & 3.4111 & 2.7765 \\
\hline 5 & 1.4740 & 1.7910 & 2.2657 & 2.9613 & 3.9647 & 3.3940 \\
\hline 6 & 1.4092 & 1.7637 & 2.3381 & 3.2409 & 4.6303 & 4.1978 \\
\hline 7 & 1.3510 & 1.7356 & 2.4113 & 3.5511 & 5.4234 & 5.2303 \\
\hline 8 & 1.3006 & 1.7081 & 2.4858 & 3.8936 & 6.3629 & 6.5458 \\
\hline 9 & 1.2581 & 1.6819 & 2.5616 & 4.2708 & 7.4720 & 8.2141 \\
\hline
\end{tabular}

We denote the solutions of problem $\left(P^{h}\right)$ and $\left(P_{\varepsilon}^{h}\right)$ by $\bar{u}^{h}$ and $\bar{u}_{\varepsilon}^{h}$ respectively. We address the reader to [7] for the details about the optimization process, [4] for theory about continuous piecewise linear approximation of Neumann control problems, and [5] for theory about approximation of Dirichlet control problems.

\section{Regularity of the solution}

Since $\bar{u} \in W^{1-1 / p, p}(\Gamma)$ for all $p<3$, we know that $\bar{u} \in H^{s}(\Gamma)$ for $s<2 / 3$. Anyway, the solution can be more regular than what is expected from the theory. Although we cannot give a proof of the fact that $\bar{u} \notin H^{s}(\Gamma)$ for $s \geq 2 / 3$, we can give some numerical evidence that this is in fact so.

We have evaluated $\left\|\bar{u}^{h}\right\|_{H^{s}(\Gamma)}$ for $h=2^{-n} \sqrt{2}, n=3, \ldots, 9$ for $s \in[0.5,1]$. To do this we have used the following norm, which is equivalent in $U^{h}$ to the usual norm in $W^{s, p}(\Gamma)$ with constants of equivalence independent of $h$ (see [6], formula (5.10)):

$$
\left\|\bar{u}^{h}\right\|_{W^{s, p}(\Gamma)}=\left(h \sum_{j=1}^{N(h)}\left|\bar{u}^{h}\left(x_{j}\right)\right|^{p}+h^{1-s p} \sum_{i \neq j} \frac{\left|\bar{u}^{h}\left(x_{j}\right)-\bar{u}^{h}\left(x_{i}\right)\right|^{p}}{\mathbf{n}(i, j)^{1+s p}}\right)^{1 / p},
$$

where $\mathbf{n}(i, j)=\min \{|j-i|, N(h)-|j-i|\}$. The results are summarized in Table 1 .

We may observe in the table that if $s>0.7$ the norm $\left\|\bar{u}^{h}\right\|_{H^{s}(\Gamma)}$ increases as $h \rightarrow 0$. Since the growth is very slow we have compared with the behavior of the approximations of a known function in the interval $(0,1)$. Let us take $r(x)=x^{1 / 6}$ in $(0,1)$. We know that $r \in H^{s}(0,1)$ for $s<2 / 3$ and $r \notin H^{s}(0,1)$ for $s \geq 2 / 3$. For $n \in \mathbb{N}$, let us define $h=1 / 2^{n}, x_{j}=j h$ for $0 \leq j \leq 2^{n}$ and $r_{h}$ the continuous piecewise linear function such that $r_{h}\left(x_{j}\right)=r\left(x_{j}\right)$. When we evaluate $\left\|r_{h}\right\|_{H^{s}(0,1)}$ (with the obvious modification $\mathbf{n}(i, j)=|i-j|$ ) we obtain the same slow growth for $s>0.7$ (see Tab. 2).

We have also observed that, if we approximate $\bar{u} \approx \bar{u}^{h^{*}}\left(h^{*}=2^{-9} \sqrt{2}\right)$, then $e_{h}=\left\|\bar{u}^{h^{*}}-\bar{u}^{h}\right\|_{L^{2}(\Gamma)} \leq 0.27 h^{0.7}$. In Figure 2 we show the double logarithmic plot of this data. The error $e_{h}=$ is reported in Table 3. Following [5], this is the expected behavior of the error if $\bar{u} \in H^{0.7}(\Gamma)$, but $\bar{u} \notin H^{s}(\Gamma)$ for $s>0.7$. 


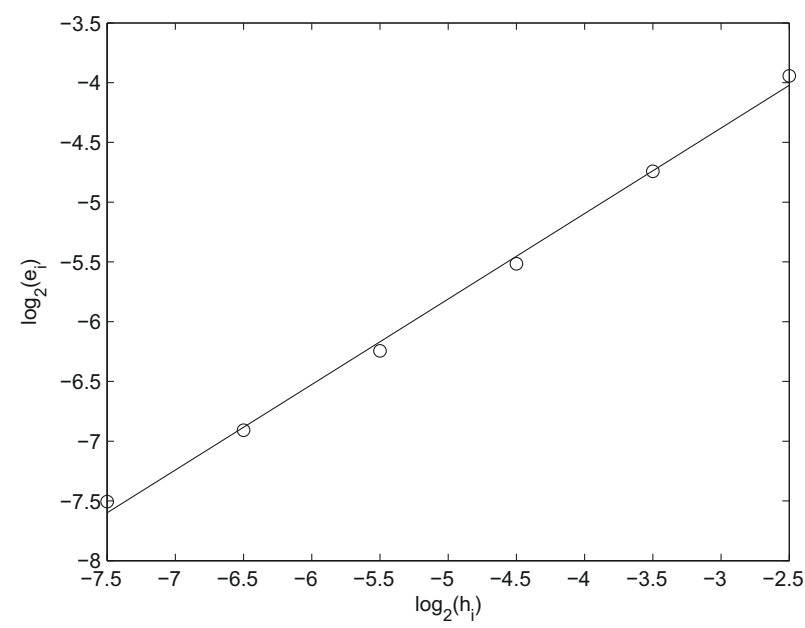

FiguRE 2. Error estimate for Dirichlet problem.

TABle 3. Error estimate for Dirichlet problem.

\begin{tabular}{|c|c|c|c|c|c|c|}
\hline$h / \sqrt{2}$ & $2^{-3}$ & $2^{-4}$ & $2^{-5}$ & $2^{-6}$ & $2^{-7}$ & $2^{-8}$ \\
\hline$e_{h}$ & 0.0650 & 0.0374 & 0.0219 & 0.0132 & 0.0083 & 0.0055 \\
\hline
\end{tabular}

\section{Checking the main estimate}

We want to check that $\left\|\bar{u}-\bar{u}_{\varepsilon}\right\|_{L^{2}(\Gamma)} \leq C \varepsilon^{0.66}$, but we do not have the exact solutions. The triangle inequality leads to

$$
\left\|\bar{u}-\bar{u}_{\varepsilon}\right\|_{L^{2}(\Gamma)} \leq\left\|\bar{u}-\bar{u}^{h}\right\|_{L^{2}(\Gamma)}+\left\|\bar{u}^{h}-\bar{u}_{\varepsilon}^{h}\right\|_{L^{2}(\Gamma)}+\left\|\bar{u}_{\varepsilon}-\bar{u}_{\varepsilon}^{h}\right\|_{L^{2}(\Gamma)} .
$$

From [5], we know that $\left\|\bar{u}-\bar{u}^{h}\right\|_{L^{2}(\Gamma)} \leq C h^{0.66}$. An easy adaptation of the proofs in [4] leads to $\left\|\bar{u}_{\varepsilon}-\bar{u}_{\varepsilon}^{h}\right\|_{L^{2}(\Gamma)} \leq$ $C h^{3 / 2} / \varepsilon$. Since we do not have the analytic solutions $\bar{u}$ and $\bar{u}_{\varepsilon}$, we will take $h^{*}=2^{-9} \sqrt{2}$ and substitute $\bar{u}$ by $\bar{u}^{h^{*}}$ and $\bar{u}_{\varepsilon}$ by $\bar{u}_{\varepsilon}^{h^{*}}$ in the estimations of the first and third addends in the right hand side of inequality (8.1). The approximate solution $y_{\bar{u}^{h}}$ is shown in Figure 1.

As we have already mentioned, we have obtained that

$$
\left\|\bar{u}-\bar{u}^{h}\right\|_{L^{2}(\Gamma)} \leq 0.27 h^{0.7}
$$

We have also solved the penalized problems for $\varepsilon=2^{-i}$ for $i=0, \ldots, 9$ and $h=2^{-n} \sqrt{2}$ for $n=3, \ldots, 9$ and we have obtained the estimate:

$$
\left\|\bar{u}_{\varepsilon}-\bar{u}_{\varepsilon}^{h}\right\|_{L^{2}(\Gamma)} \leq \frac{0.07}{\varepsilon} h^{1.85} .
$$

So, if we choose $h$ very small, we can expect that the second term in the right hand side of inequality (8.1) is the dominant term in the sum. We are going to fix $h^{*}=2^{-9} \sqrt{2}$ and evaluate $\left\|\bar{u}^{h^{*}}-\bar{u}_{\varepsilon}^{h^{*}}\right\|_{L^{2}(\Gamma)}$ for different values of $\varepsilon$. Since $h^{*}$ is very small (the mesh contains approximately $5.2 \times 10^{5}$ triangles, $2.6 \times 10^{5}$ nodes and 2048 boundary nodes, that is the best we can do with MATLAB on a PC), we can expect that the error due to the numerical approximation is significatively smaller than the error due to the penalization, at least while $\varepsilon$ is not very small. Therefore we can approximate:

$$
\left\|\bar{u}-\bar{u}_{\varepsilon}\right\|_{L^{2}(\Gamma)} \approx\left\|\bar{u}^{h^{*}}-\bar{u}_{\varepsilon}^{h^{*}}\right\|_{L^{2}(\Gamma)} .
$$


TABLE 4. Order of convergence for the solutions of the penalized problems.

\begin{tabular}{|c|c|c|}
\hline$\varepsilon_{i}$ & $e_{i}$ & $o_{i}$ \\
\hline 1 & 0.1545 & 0.35 \\
\hline $2^{-1}$ & 0.1209 & 0.46 \\
\hline $2^{-2}$ & 0.0877 & 0.57 \\
\hline \hline $2^{-3}$ & 0.0592 & 0.65 \\
\hline $2^{-4}$ & 0.0378 & 0.69 \\
\hline $2^{-5}$ & 0.0235 & 0.68 \\
\hline $2^{-6}$ & 0.0146 & 0.62 \\
\hline \hline $2^{-7}$ & 0.0095 & 0.51 \\
\hline $2^{-8}$ & 0.0067 & 0.41 \\
\hline $2^{-9}$ & 0.0050 & - \\
\hline
\end{tabular}

To evaluate the order of convergence as $\varepsilon \rightarrow 0$, we solve the penalized problem for $\varepsilon_{i}=2^{-i}, i=0, \ldots, 9$. For each $i$, we define the experimental error as

$$
e_{i}=\left\|u^{h^{*}}-u_{\varepsilon_{i}}^{h^{*}}\right\|_{L^{2}(\Gamma)},
$$

and the experimental order of convergence between two consecutive values of $\varepsilon_{i}$ as

$$
o_{i}=\frac{\log \left(e_{i+1}\right)-\log \left(e_{i}\right)}{\log \left(\varepsilon_{i+1}\right)-\log \left(\varepsilon_{i}\right)} .
$$

The experimental results are summarized in Table 4 . Observe that for $i=3, \ldots, 6$ the experimental order of convergence is quite close to the order of convergence expected from the theory: $o=0.66$. For small values of $\varepsilon$ the error due to the penalization is overlapped with the error due to the numerical approximation. That is the reason for $o_{7}$ and $o_{8}$ to be smaller than expected. You may also observe that $o_{0}, o_{1}$ and $o_{2}$ are also smaller than the value predicted by the theory. This is the usual behavior with penalized problems: the ratio $o_{i}$ becomes a sharp estimate of the order of convergence only asymptotically. To understand what is happening, think of this naive example about penalization of PDEs: Consider the unit ball in $D$ in $\mathbb{R}^{2}$, denote by $\partial D$ its boundary and by $(r, \theta)$ the polar coordinates. We can solve exactly the problems

$$
\begin{gathered}
-\Delta y=0 \text { on } D, y=\sin \theta \text { on } \partial D, \\
-\Delta y_{\varepsilon}=0 \text { on } D, \varepsilon \partial_{\nu} y_{\varepsilon}+y_{\varepsilon}=\sin \theta \text { on } \partial D .
\end{gathered}
$$

We obtain $y=r \sin \theta$ and $y_{\varepsilon}=y /(1+\varepsilon)$. We can compute exactly

$$
\left\|y-y_{\varepsilon}\right\|_{L^{2}(D)}=\frac{\pi}{4} \frac{\varepsilon}{1+\varepsilon} .
$$

So the estimate $\left\|y-y_{\varepsilon}\right\|_{L^{2}(D)} \leq \frac{\pi}{4} \varepsilon$ is true for all $\varepsilon>0$, but it is very rough for big values of $\varepsilon$. The experimental orders of convergence corresponding to the values of $\varepsilon=2^{-i}, i=0, \ldots, 9$ are $(0.58,0.74,0.85$, $0.92,0.96,0.98,0.99,0.99,1.00)$. The order of convergence is close to 1.00 (the theoretical order of convergence) only for $\varepsilon \leq 2^{-4}$.

In Figure 3 we can see a double logarithmic plot of the data in Table 4 versus the line corresponding to an order of convergence $o=0.67$. This number is obtained from a linear regression from the central values of the figure. The estimate we obtain for $\varepsilon_{i}=2^{-i}, i=3, \ldots, 6$ is

$$
\left\|\bar{u}-\bar{u}_{\varepsilon}\right\|_{L^{2}(\Gamma)} \leq 0.24 \varepsilon^{0.67}
$$




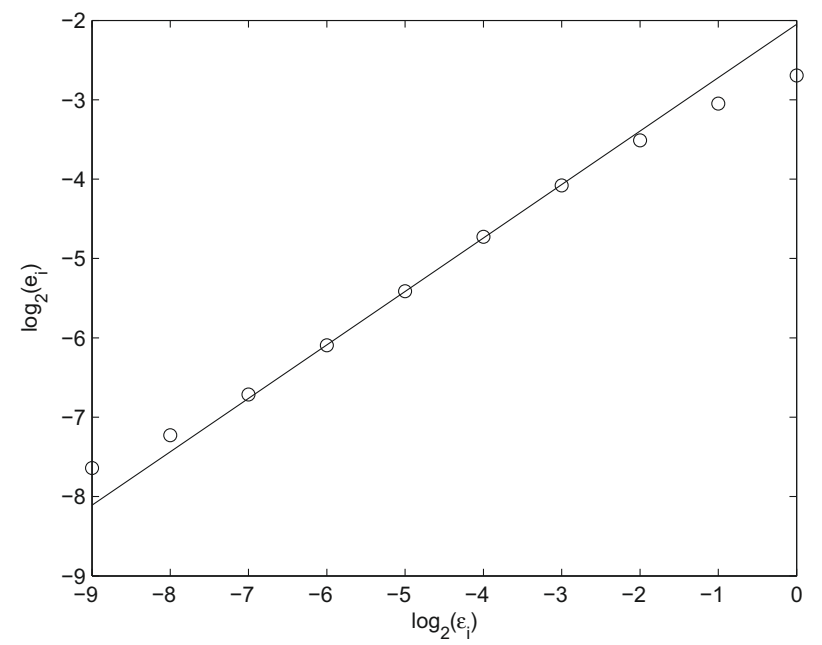

Figure 3. Dots: $\log _{2}\left(\varepsilon_{i}\right)$ vs. $\log _{2}\left(e_{i}\right)$. Solid: $\log _{2}\left(\varepsilon_{i}\right)$ vs. $0.24+0.67 \log _{2}\left(\varepsilon_{i}\right)$.

\section{Appendix A: Regularity Results for solutions of ELliptic PARTial Differential EQUATIONS IN CONVEX POLYGONS}

Let us recall that throughout this section $C(M)>0$ denotes various constants depending on $M$ where $\left\|a_{0}\right\|_{L^{p}(\Omega)} \leq M$.

In order to prove Theorem 2.2, we need some regularity results for the equation

$$
-\Delta y_{\varepsilon}+a_{0}(x) y_{\varepsilon}=0 \text { in } \Omega, \quad \varepsilon \partial_{\nu} y_{\varepsilon}+y_{\varepsilon}=u \text { on } \Gamma
$$

where $u \in H^{s}(\Gamma)$, with $-1 \leq s \leq 1 / 2$, and for the equation

$$
-\Delta y+a_{0}(x) y=0 \text { in } \Omega, y=\eta \text { on } \Gamma,
$$

where $\eta \in H^{s}(\Gamma)$, with $-1 / 2 \leq s \leq 1$.

Before that we need some trace regularity results that we state below.

\section{A.1. Trace regularity results}

For general trace results in Lipschitz domains, we refer to [10]. To our best knowledge, it has not been proved that the trace of a function in $H^{3 / 2}(\Omega)$ is in $H^{1}(\Gamma)$. Nevertheless, this is known to be true for harmonic functions in Lipschitz domains (see $[13,14]$ ). The next lemma shows how to extend this result for our purposes.

Lemma A.1. Let $\Omega$ be an open convex bounded domain of $\mathbb{R}^{n}, n \geq 1$ (here $\Omega$ is not necessarily a polygonal domain). If $\Delta z \in L^{2}(\Omega)$ and $z \in H^{3 / 2}(\Omega)$, then $\left.z\right|_{\Gamma} \in H^{1}(\Gamma)$ and $\partial_{\nu} z \in L^{2}(\Gamma)$, and we have the estimate

$$
\left\|\left.z\right|_{\Gamma}\right\|_{H^{1}(\Gamma)}+\left\|\partial_{\nu} z\right\|_{L^{2}(\Gamma)} \leq C\left(\|\Delta z\|_{L^{2}(\Omega)}+\|z\|_{H^{3 / 2}(\Omega)}\right) .
$$

Proof. Let $z_{0}, z_{1} \in H^{1}(\Omega)$ be respectively the solutions of

$$
\Delta z_{0}=0 \text { in } \Omega, \quad z_{0}=\left.z\right|_{\Gamma} \text { on } \Gamma
$$

and

$$
\Delta z_{1}=\Delta z \text { in } \Omega, \quad z_{1}=0 \text { on } \Gamma .
$$


It is clear that $z=z_{0}+z_{1}$ and $\left.z\right|_{\Gamma}=\left.z_{0}\right|_{\Gamma}$. From [11] it follows that $z_{1}$ belongs to $H^{2}(\Omega)$ and that

$$
\left\|z_{1}\right\|_{H^{2}(\Omega)} \leq C\|\Delta z\|_{L^{2}(\Omega)} .
$$

Since $z_{0}=z-z_{1}$ and $z \in H^{3 / 2}(\Omega)$, we can claim that $z_{0}$ belongs to $H^{3 / 2}(\Omega)$. From [14], Theorem 5.6 and Corollary 5.7, and the previous estimate, it follows that

$$
\begin{aligned}
\left\|\left.z_{0}\right|_{\Gamma}\right\|_{H^{1}(\Gamma)}+\left\|\partial_{\nu} z_{0}\right\|_{L^{2}(\Gamma)} \leq C\left\|z_{0}\right\|_{H^{3 / 2}(\Omega)} & \leq\left\|z_{1}\right\|_{H^{3 / 2}(\Omega)}+\|z\|_{H^{3 / 2}(\Omega)} \\
& \leq\left\|z_{1}\right\|_{H^{2}(\Omega)}+\|z\|_{H^{3 / 2}(\Omega)} \leq C\left(\|\Delta z\|_{L^{2}(\Omega)}+\|z\|_{H^{3 / 2}(\Omega)}\right) .
\end{aligned}
$$

Therefore the estimate of $\left.z\right|_{\Gamma}$ in $H^{1}(\Gamma)$ is established. Due to Lemma A.2 below, we also have

$$
\left\|\partial_{\nu} z_{1}\right\|_{L^{2}(\Gamma)} \leq C\|\Delta z\|_{L^{2}(\Omega)} .
$$

The proof is complete.

It is also known that in a Lipschitz domain, the normal derivative of a function in $C^{\infty}(\Omega)$ does not necessarily belongs to $H^{1 / 2}(\Gamma)$ (think of $y(x)=|x|^{2}$ on the unit square $\Omega=(0,1)^{2}$ ). With some extra regularity and a condition on the trace of the function, we get the following result.

Lemma A.2. Let $2 \leq q \leq p$. If $g \in L^{q}(\Omega)$, then the solution $z$ to equation

$$
-\Delta z+a_{0}(x) z=g \text { in } \Omega, \quad z=0 \text { on } \Gamma,
$$

belongs to $W^{2, q}(\Omega)$ and obeys

$$
\|z\|_{W^{2, q}(\Omega)}+\left\|\partial_{\nu} z\right\|_{W^{1-1 / q, q}(\Gamma)} \leq C(M)\|g\|_{L^{q}(\Omega)} .
$$

Proof. Let us first give the proof in the case $q=2$. Since $a_{0} \geq 0$, from the results in Stampacchia [17] we deduce that $z \in H_{0}^{1}(\Omega) \cap L^{\infty}(\Omega)$ and there exists a constant $C>0$ independent of $a_{0}$ such that

$$
\|z\|_{H^{1}(\Omega)}+\|z\|_{L^{\infty}(\Omega)} \leq C\|g\|_{L^{2}(\Omega)} .
$$

So we have $a_{0} z \in L^{2}(\Omega)$ and

$$
\left\|a_{0} z\right\|_{L^{2}(\Omega)} \leq\left\|a_{0}\right\|_{L^{p}(\Omega)}\|z\|_{L^{\infty}(\Omega)} \leq M \cdot C\|g\|_{L^{2}(\Omega)} .
$$

Following [11], we know that $z \in H^{2}(\Omega)$ and there exists a constant $C>0$ independent of $a_{0}$ such that

$$
\|z\|_{H^{2}(\Omega)} \leq C\left\|g-a_{0} z\right\|_{L^{2}(\Omega)} \leq C(M)\|g\|_{L^{2}(\Omega)} .
$$

Since $\Omega$ is a polygonal domain, we have $\bar{\Omega}=\operatorname{co}\left\{S_{j} \mid 0 \leq j \leq N_{S}\right\}$, where 'co' stands for the convex hull and $\left(S_{j}\right)_{0 \leq j \leq N_{S}}$ are the vertices of the polygonal domain. We assume that the vertices are numbered counterclockwise and that $S_{0}=S_{N_{S}}$. With this convention, the boundary $\Gamma$ is the union of the edges $\Gamma_{j}=\left[S_{j-1}, S_{j}\right]$, where $1 \leq j \leq N_{S}$. The trace of $\nabla z$ on $\Gamma$ belongs to $\left(H^{1 / 2}(\Gamma)\right)^{2}$. Thus the trace of $\nabla z$ on $\Gamma_{j}$ belongs to $\left(H^{1 / 2}\left(\Gamma_{j}\right)\right)^{2}$ and $\partial_{\nu} z \in H^{1 / 2}\left(\Gamma_{j}\right)$. Moreover we have the estimate

$$
\left\|\partial_{\nu} z\right\|_{H^{1 / 2}\left(\Gamma_{j}\right)} \leq C(M)\|g\|_{L^{2}(\Omega)} .
$$

To show that $\partial_{\nu} z$ belongs to $H^{1 / 2}(\Gamma)$ we have to analyze what happens at the corners $S_{j}=\Gamma_{j} \cap \Gamma_{j+1}$. For that, we can parametrize the edge $\Gamma_{j+1}$ by setting $x_{j}(\sigma)=S_{j}+\frac{\sigma}{\left|\Gamma_{j+1}\right|}\left(S_{j+1}-S_{j}\right)$ with $0 \leq \sigma \leq\left|\Gamma_{j+1}\right|$, and the points of $\Gamma_{j}$ by $x_{j}(-\sigma)=S_{j}-\frac{\sigma}{\left|\Gamma_{j}\right|}\left(S_{j}-S_{j-1}\right)$ with $0 \leq \sigma \leq\left|\Gamma_{j}\right|$. For $0 \leq \sigma \leq \delta_{j}=\min \left\{\left|\Gamma_{j}\right|,\left|\Gamma_{j+1}\right|\right\}$, 
$x_{j}(\sigma) \in \Gamma_{j+1}, x_{j}(-\sigma) \in \Gamma_{j}$ and $\left|x_{j}(\sigma)-S_{j}\right|=\left|x_{j}(-\sigma)-S_{j}\right|=\sigma$. According to Theorem 1.5.2.3.c in [11], to prove that $\partial_{\nu} z \in H^{1 / 2}\left(\Gamma_{j} \cup \Gamma_{j+1}\right)$, we have to show that

$$
\int_{0}^{\delta_{j}} \frac{\left|\partial_{\nu} z\left(x_{j}(\sigma)\right)-\partial_{\nu} z\left(x_{j}(-\sigma)\right)\right|^{2}}{\sigma} \mathrm{d} \sigma<+\infty
$$

First notice that $\|z\|_{H^{2}(\Omega)} \leq C(M)\|g\|_{L^{2}(\Omega)}$. Since $z \in H^{2}(\Omega)$, then $\nabla z \in\left(H^{1}(\Omega)\right)^{2}$, and the usual trace theorem says that $\partial_{i} z \in H^{1 / 2}(\Gamma)$ for $i=1,2$. As is shown in the first part of the proof of Theorem 1.5.2.3.c in [11], this implies that

$$
\int_{0}^{\delta_{j}} \frac{\left|\partial_{i} z\left(x_{j}(\sigma)\right)-\partial_{i} z\left(x_{j}(-\sigma)\right)\right|^{2}}{\sigma} \mathrm{d} \sigma<+\infty
$$

for $i=1,2$. We are going to transform the integral involving the normal derivative into a combinations of integrals involving the partial derivatives. To do that, without loss of generality, we can suppose that $\Gamma_{j}$ is on the negative part of the $x$ axis, $S_{j}$ is at the origin and $\Gamma_{j+1} \subset\left\{\left(-\sigma n_{2}, \sigma n_{1}\right) \mid 0 \leq \sigma\right\}$, so that $\nu_{j}=(0,-1)$ and $\nu_{j+1}=\left(n_{1}, n_{2}\right)$, where $n_{1}>0$ and $n_{1}^{2}+n_{2}^{2}=1$. Therefore

$$
\int_{0}^{\delta_{j}} \frac{\left|\partial_{\nu} z\left(x_{j}(\sigma)\right)-\partial_{\nu} z\left(x_{j}(-\sigma)\right)\right|^{2}}{\sigma} \mathrm{d} \sigma=\int_{0}^{\delta_{j}} \frac{\left|n_{1} \partial_{1} z\left(x_{j}(\sigma)\right)+n_{2} \partial_{2} z\left(x_{j}(\sigma)\right)+\partial_{2} z\left(x_{j}(-\sigma)\right)\right|^{2}}{\sigma} \mathrm{d} \sigma .
$$

Since $z=0$ on $\Gamma$, the tangential derivative is 0 , i.e., $\partial_{1} z\left(x_{j}(-\sigma)\right)=0$ and $-n_{2} \partial_{1} z\left(x_{j}(\sigma)\right)+n_{1} \partial_{2} z\left(x_{j}(\sigma)\right)=0$. If we take $\gamma=-\left(1+n_{2}\right) / n_{1}$ and $\beta=n_{2} \gamma-n_{1}$ and if we observe that the linear combination $\gamma\left(-n_{2} \partial_{1} z\left(x_{j}(\sigma)\right)+\right.$ $\left.n_{1} \partial_{2} z\left(x_{j}(\sigma)\right)\right)+\beta \partial_{1} z\left(x_{j}(-\sigma)\right)$ is equal to zero, the RHS of (A.4) is bounded by

$$
2 \beta^{2} \int_{0}^{\delta_{j}} \frac{\left|\partial_{1} z\left(x_{j}(\sigma)\right)-\partial_{1} z\left(x_{j}(-\sigma)\right)\right|^{2}}{\sigma} \mathrm{d} \sigma+2 \int_{0}^{\delta_{j}} \frac{\left|\partial_{2} z\left(x_{j}(\sigma)\right)-\partial_{2} z\left(x_{j}(-\sigma)\right)\right|^{2}}{\sigma} \mathrm{d} \sigma<+\infty .
$$

Making the same analysis for each corner, we have proved estimate (A.3) for $q=2$. Let us now consider the case when $2<q \leq p$. If $g \in L^{q}(\Omega)$, then $z \in W^{2, q}(\Omega)$,

$$
\|z\|_{W^{2, q}(\Omega)} \leq C(M)\|g\|_{L^{q}(\Omega)}
$$

and therefore $\nabla z \in\left(W^{1, q}(\Omega)\right)^{2}$. From a usual trace theorem in Lipschitz domains it follows that $\partial_{i} z \in$ $W^{1-1 / q, q}(\Gamma)$ for $i=1,2$. This implies that the traces of the partial derivatives are continuous at the corners of the domain (Thm. 1.5.2.3.b in [11]). Moreover we have the estimate

$$
\left\|\partial_{\nu} z\right\|_{W^{1-1 / q, q}\left(\Gamma_{j}\right)} \leq C(M)\|g\|_{L^{q}(\Omega)} .
$$

Since the normal vector to $\Gamma$ is a discontinuous field at the corners of $\Omega$, we cannot deduce that $\partial_{\nu} z$ belongs to $W^{1-1 / q, q}(\Gamma)$ from the fact that $z$ belongs to $W^{2, q}(\Omega)$. As in the case when $q=2$, the problem arises at the corners $S_{j}$. According to Theorem 1.5.2.3.b in [11] to prove that $\partial_{\nu} z \in W^{1-1 / q, q}\left(\Gamma_{j} \cup \Gamma_{j+1}\right)$, we have to show that $\partial_{\nu} z$ is continuous at the corner $S_{j}$.

Using the notation introduced in the case $q=2$, we obtain

$$
\left|\partial_{\nu} z\left(x_{j}(\sigma)\right)-\partial_{\nu} z\left(x_{j}(-\sigma)\right)\right| \leq \beta\left|\partial_{1} z\left(x_{j}(\sigma)\right)-\partial_{1} z\left(x_{j}(-\sigma)\right)\right|+\left|\partial_{2} z\left(x_{j}(\sigma)\right)-\partial_{2} z\left(x_{j}(-\sigma)\right)\right|
$$

and the continuity at $S_{j}$ of the normal derivative follows from the continuity of the traces of the partial derivatives. Moreover, the continuity of $\partial_{\nu} z$ being proved at the corner $S_{j}$, we have

$$
\left\|\partial_{\nu} z\right\|_{W^{1-1 / q, q}\left(\Gamma_{j} \cup \Gamma_{j+1}\right)} \leq C\left(\left\|\partial_{\nu} z\right\|_{W^{1-1 / q, q}\left(\Gamma_{j}\right)}+\left\|\partial_{\nu} z\right\|_{W^{1-1 / q, q}\left(\Gamma_{j+1}\right)}\right) \leq C(M)\|g\|_{L^{q}(\Omega)} .
$$

This estimate being true for each node $S_{j}, 0 \leq j \leq N_{S}$, estimate (A.3) is proved. 
We can now obtain results analogous to those in Lemma A.1 for functions whose Laplacian is not so regular, but instead satisfy homogeneous boundary conditions.

Lemma A.3. Let $g$ belong to $\left(H^{1 / 2}(\Omega)\right)^{\prime}$. Then, the solution $z$ to equation

$$
-\Delta z+a_{0}(x) z=g \text { in } \Omega, \quad z=0 \text { on } \Gamma,
$$

belongs to $H^{3 / 2}(\Omega)$ and obeys

$$
\left\|\partial_{\nu} z\right\|_{L^{2}(\Gamma)} \leq C(M)\|g\|_{\left(H^{1 / 2}(\Omega)\right)^{\prime}} .
$$

Proof. The proof is very similar to the one in [16] (Thm. A.1 and Cor. A.1). Observe that when $g \in\left(H^{1 / 2}(\Omega)\right)^{\prime}$ the solution $z$ is defined by the transposition method. (The result of the lemma uses interpolation theory, and for interpolation results in Lipschitz domains, we refer to [14].)

Lemma A.4. Let $g$ belong to $\left(H^{1 / 2}(\Omega)\right)^{\prime}$. Then, the solution $z$ to equation

$$
-\Delta z+a_{0}(x) z=g \text { in } \Omega, \quad \partial_{\nu} z+z=0 \text { on } \Gamma,
$$

belongs to $H^{3 / 2}(\Omega)$ and obeys

$$
\left\|\left.z\right|_{\Gamma}\right\|_{H^{1}(\Gamma)} \leq C(M)\|g\|_{\left(H^{1 / 2}(\Omega)\right)^{\prime}} .
$$

Proof. The proof also follows by interpolation. If $g \in\left(H^{1}(\Omega)\right)^{\prime}$, then by the Lax-Milgram Theorem and the usual trace theory, we know that $z \in H^{1}(\Omega),\left.z\right|_{\Gamma} \in H^{1 / 2}(\Gamma)$ and there exists a constant $C>0$ independent of $a_{0}$ such that

$$
\left\|\left.z\right|_{\Gamma}\right\|_{H^{1 / 2}(\Gamma)} \leq C\|g\|_{\left(H^{1}(\Omega)\right)^{\prime}} .
$$

In particular, for each side $\Gamma_{j}$ it is obvious that

$$
\left\|\left.z\right|_{\Gamma_{j}}\right\|_{H^{1 / 2}\left(\Gamma_{j}\right)} \leq C\|g\|_{\left(H^{1}(\Omega)\right)^{\prime}} .
$$

If $g \in L^{2}(\Omega)$, then $z \in H^{2}(\Omega)$ (cf. Thm. 3.2.3.1 in [11]). Using Lemma A.1, we know that $\left.z\right|_{\Gamma} \in H^{1}(\Gamma)$ and

$$
\left\|\left.z\right|_{\Gamma}\right\|_{H^{1}(\Gamma)} \leq C(M)\|g\|_{L^{2}(\Omega)} .
$$

According to Theorem 1.5.2.8 in [11], $\left.z\right|_{\Gamma_{j}} \in H^{3 / 2}\left(\Gamma_{j}\right)$ for each side $\Gamma_{j}$ and

$$
\left\|\left.z\right|_{\Gamma_{j}}\right\|_{H^{3 / 2}\left(\Gamma_{j}\right)} \leq C(M)\|g\|_{L^{2}(\Omega)} .
$$

For $g \in\left(H^{1 / 2}(\Omega)\right)^{\prime}$, by interpolation between estimates (A.8) and (A.10), we obtain that $\left.z\right|_{\Gamma_{j}} \in H^{1}\left(\Gamma_{j}\right)$ for each side $\Gamma_{j}$ and

$$
\left\|\left.z\right|_{\Gamma_{j}}\right\|_{H^{1}\left(\Gamma_{j}\right)} \leq C(M)\|g\|_{\left(H^{1 / 2}(\Omega)\right)^{\prime}} .
$$

Using estimates (A.7) and (A.9), we know that $\left.z\right|_{\Gamma} \in H^{3 / 4}(\Gamma)$. This implies that $\left.z\right|_{\Gamma}$ is continuous at the corners and thus $\left.z\right|_{\Gamma} \in H^{1}(\Gamma)$. To prove the last point we can imagine (by using a Lipschitz parametrization) that $z$ is defined in an interval, divided into a finite number of subintervals $\left(x_{j}, x_{j+1}\right),\left.z\right|_{\Gamma}$ belongs to $H^{1}\left(x_{j}, x_{j+1}\right)$ and it is continuous in the whole interval. Then it is well known ( $c f$. Thm. 5.1 in [8]) that $\left.z\right|_{\Gamma}$ belongs to $H^{1}(\Gamma)$.

Finally, estimate (A.6) follows adding up estimates (A.11) for all the sides $\Gamma_{j}$. 


\section{A.2. Regularity results for equation (A.1)}

In the case when $-1 / 2 \leq s$, thanks to the Lax-Milgram Theorem, it can be shown that equation (A.1) admits a unique solution $y$ in $H^{1}(\Omega)$. When $-1 \leq s<-1 / 2$, solutions to equation (A.1) may be defined by the transposition method. For that we consider the following variational problem

Find $y_{\varepsilon} \in L^{2}(\Omega)$ and $\lambda_{\varepsilon} \in H^{-1 / 2}(\Gamma)$ such that

$$
\int_{\Omega} y_{\varepsilon} g \mathrm{~d} x+\frac{1}{\varepsilon}\left\langle\lambda_{\varepsilon}, v\right\rangle_{H^{-1 / 2}(\Gamma), H^{1 / 2}(\Gamma)}=\frac{1}{\varepsilon}\langle u, z\rangle_{H^{s}(\Gamma), H^{-s}(\Gamma)},
$$

for all $g \in L^{2}(\Omega)$ and all $v \in H^{1 / 2}(\Gamma)$, where $z$ is the solution to

$$
-\Delta z+a_{0}(x) z=g \text { in } \Omega, \varepsilon \partial_{\nu} z+z=v \text { on } \Gamma \text {. }
$$

Notice that for $s \geq-1 / 2$, if $y_{\varepsilon}$ is a solution to (A.1), then $\left(y_{\varepsilon},\left.y_{\varepsilon}\right|_{\Gamma}\right)$ is a solution to (A.12). Since $\lambda_{\varepsilon}$ plays the role of the trace of $y_{\varepsilon}$ in the case when $u$ is regular, we will make an abuse of notation by replacing $\lambda_{\varepsilon}$ by $\left.y_{\varepsilon}\right|_{\Gamma}$.

Now we are going to give regularity results for the solution to equation (A.1) in the case when $\varepsilon=1$. The dependence of the solution with respect to $\varepsilon$, when $0<\varepsilon<1$, will be studied in section A.4, and precise results are stated in Theorem 2.2 .

Lemma A.5. Take $\varepsilon=1$ and let $-1 \leq s \leq 1 / 2$. If $u \in H^{s}(\Gamma)$, then the solution $y$ to equation (A.1) belongs to $H^{s+3 / 2}(\Omega)$, and there exists a constant $C(M)>0$ such that

$$
\|y\|_{H^{s+3 / 2}(\Omega)} \leq C(M)\|u\|_{H^{s}(\Gamma)} .
$$

Moreover, for $-1 \leq s \leq 0,\left.y\right|_{\Gamma} \in H^{s+1}(\Gamma)$ and

$$
\left\|\left.y\right|_{\Gamma}\right\|_{H^{s+1}(\Gamma)} \leq C(M)\|u\|_{H^{s}(\Gamma)} .
$$

Proof. For $s=-1 / 2$, as already mentioned before, the existence of a unique solution $y$ in $H^{1}(\Omega)$ and estimate (A.13) follow from the Lax-Milgram Theorem. Estimate (A.14) for $s=-1 / 2$ follows from the usual trace theorem in $H^{1}(\Omega)$. The constant in this case is also independent of $M$.

For $s=1 / 2$, we know that $-\Delta y=-a_{0} y \in L^{2}(\Omega)$, because $a_{0} \in L^{p}(\Omega)$ and $y \in H^{1}(\Omega) \subset L^{q}(\Omega)$ for all $q<\infty$. Thus, the $H^{2}(\Omega)$ regularity of $y$ and estimate (A.13) follow from Corollary 4.4.4.14 in Grisvard [11].

Notice that $\left.y\right|_{\Gamma}$, the trace of $y$, does not necessarily belong to $H^{3 / 2}(\Gamma)$ (think again of $y(x)=|x|^{2}$ in the unit square $\left.\Omega=(0,1)^{2}\right)$, so we cannot state estimate (A.14) for $s=1 / 2$.

For $-1 / 2 \leq s \leq 1 / 2$, estimate (A.13) is obtained by interpolation.

Now, we can prove (A.14) for $s=0$. Since we have already proved estimate (A.13) for $s=0$, we know that $y \in H^{3 / 2}(\Omega)$ and $-\Delta y=-a_{0} y \in L^{2}(\Omega)$. Thus estimate (A.14) follows from Lemma A.1 and estimate (A.13) for $s=0$.

Let us consider the case when $s=-1$. Let us first notice that the uniqueness result is obvious. Indeed, if $(y, \lambda) \in L^{2}(\Omega) \times H^{-1 / 2}(\Gamma)$ is a solution to problem (A.12) corresponding to $u=0$, then by taking $g=y$ and $v=0$, we first obtain that $y=0$. If $y=0$ and $u=0$ in (A.12), it is clear that $\lambda=0$.

To prove the existence of a solution, we proceed by approximation. Let $u$ be in $H^{-1}(\Gamma)$, and let $\left\{u_{k}\right\}$ be a sequence in $L^{2}(\Gamma)$ converging to $u$ in $H^{-1}(\Gamma)$. For every $k$, let $y_{k} \in H^{3 / 2}(\Omega)$ be the solution to equation (A.1) corresponding to $u_{k}$. Due to Lemma A.1, $\left.y_{k}\right|_{\Gamma}$ belongs to $H^{1}(\Gamma)$.

For all $g \in L^{2}(\Omega)$ and all $v \in H^{1 / 2}(\Gamma)$, we consider the solution $z \in H^{2}(\Omega)$ to the equation

$$
-\Delta z+a_{0} z=g \text { in } \Omega, \partial_{\nu} z+z=v \text { on } \Gamma \text {. }
$$


We easily verify that $y_{k}$ and $z$ obey

$$
\int_{\Omega} y_{k} g \mathrm{~d} x+\int_{\Gamma} y_{k} v \mathrm{~d} \sigma(x)=\int_{\Gamma} u_{k} z \mathrm{~d} \sigma(x) .
$$

First take $v=0$. From identity (A.15) when $v=0$, and from estimate (A.6), we deduce

$$
\begin{aligned}
& \left\|y_{k}\right\|_{H^{1 / 2}(\Omega)}=\sup _{\|g\|_{\left(H^{1 / 2}(\Omega)\right)^{\prime}}=1} \int_{\Omega} y_{k} g \mathrm{~d} x=\sup _{\|g\|_{\left(H^{1 / 2}(\Omega)\right)^{\prime}}=1}\left\langle u_{k}, z\right\rangle_{H^{-1}(\Gamma), H^{1}(\Gamma)} \\
& \leq \sup _{\|g\|_{\left(H^{1 / 2}(\Omega)\right)^{\prime}}=1}\left\|u_{k}\right\|_{H^{-1}(\Gamma)}\|z\|_{H^{1}(\Gamma)} \leq C(M)\left\|u_{k}\right\|_{H^{-1}(\Gamma)} .
\end{aligned}
$$

To estimate $\left.y_{k}\right|_{\Gamma}$, we take $g=0$ in (A.15). Estimate (A.14) for $s=0$ (already proved above), implies that $\left\|\left.z\right|_{\Gamma}\right\|_{H^{1}(\Gamma)} \leq C(M)\|v\|_{L^{2}(\Gamma)}$, and we have

$$
\begin{aligned}
\left\|\left.y_{k}\right|_{\Gamma}\right\|_{L^{2}(\Gamma)} & =\sup _{\|v\|_{L^{2}(\Gamma)}=1} \int_{\Gamma} y_{k} v \mathrm{~d} \sigma(x) \\
& =\sup _{\|v\|_{L^{2}(\Gamma)}=1}\left\langle u_{k},\left.z\right|_{\Gamma}\right\rangle_{H^{-1}(\Gamma), H^{1}(\Gamma)} \leq \sup _{\|v\|_{L^{2}(\Gamma)}=1}\left\|u_{k}\right\|_{H^{-1}(\Gamma)}\left\|\left.z\right|_{\Gamma}\right\|_{H^{1}(\Gamma)} \leq C(M)\left\|u_{k}\right\|_{H^{-1}(\Gamma)}
\end{aligned}
$$

Notice that we also have

$$
\left\|y_{k}-y_{\ell}\right\|_{H^{1 / 2}(\Omega)}+\left\|y_{k}-y_{\ell}\right\|_{L^{2}(\Gamma)} \leq C(M)\left\|u_{k}-u_{\ell}\right\|_{H^{-1}(\Gamma)}
$$

for all $k$ and all $\ell$.

From the previous estimates it follows that $\left\{y_{k}\right\}$ converges to some $y$ in $H^{1 / 2}(\Omega)$, and $\left\{\left.y_{k}\right|_{\Gamma}\right\}$ converges to some $\lambda$ in $L^{2}(\Gamma)$. By passing to the limit in (A.15), we easily verify that $(y, \lambda)$ is a solution of (A.12). Thus $\left.y\right|_{\Gamma}=\lambda$. Moreover, we have

$$
\|y\|_{H^{1 / 2}(\Omega)} \leq C(M)\|u\|_{H^{-1}(\Gamma)} \quad \text { and } \quad\left\|\left.y\right|_{\Gamma}\right\|_{L^{2}(\Gamma)} \leq C(M)\|u\|_{H^{-1}(\Gamma)} .
$$

The remaining cases are obtained by interpolation.

\section{A.3. Regularity results for solutions to equation (A.2)}

Now, we are going to study the Dirichlet problem (A.2) when $\eta \in H^{s}(\Gamma)$, with $-1 / 2 \leq s \leq 1$.

If $\eta \in H^{1 / 2}(\Gamma)$, the existence of a unique solution $y \in H^{1}(\Omega)$ to equation (A.2) satisfying

$$
\|y\|_{H^{1}(\Omega)} \leq C\|\eta\|_{H^{1 / 2}(\Gamma)},
$$

is classical, and it can be shown by using the fact that the trace mapping $\gamma_{0}$ is a bounded and surjective operator from $H^{1}(\Omega)$ to $H^{1 / 2}(\Gamma)$.

If $0 \leq s<1 / 2$, then solutions to equation (A.2) can be defined by the transposition method in the following way. For that we consider the following variational problem

Find $y \in H^{1 / 2}(\Omega)$ and $\chi \in H^{-1}(\Gamma)$ such that

$\int_{\Omega} y g \mathrm{~d} x-\langle\chi, v\rangle_{H^{-1}(\Gamma), H^{1}(\Gamma)}=-\int_{\Gamma} \partial_{\nu} z \eta \mathrm{d} \sigma(x)$,

for all $g \in L^{2}(\Omega)$ and all $v \in H^{1}(\Gamma)$, where $z$ is the solution to

$-\Delta z+a_{0} z=g$ in $\Omega, z=v$ on $\Gamma$. 
Due to Lemma A.6 for $s=1, \partial_{\nu} z \in L^{2}(\Gamma)$ and the definition makes sense. Notice that $\chi$ is going to play the role of the normal derivative of $y$ for regular problems, so we make an abuse of notation and denote $\partial_{\nu} y:=\chi$.

Solutions to equation (A.2) can also be defined for $-1 / 2 \leq s<0$. For that we consider the following variational problem

Find $y \in L^{2}(\Omega)$ such that

$$
\begin{aligned}
& \int_{\Omega} y g \mathrm{~d} x=-\int_{\Gamma} \partial_{\nu} z \eta \mathrm{d} \sigma(x), \\
& \text { for all } g \in L^{2}(\Omega), \text { where } z \text { is the solution to } \\
& -\Delta z+a_{0} z=g \text { in } \Omega, z=0 \text { on } \Gamma .
\end{aligned}
$$

Lemma A.6. If $\eta \in H^{s}(\Gamma)$ with $-1 / 2 \leq s \leq 1$, then there exists a unique $y \in H^{s+1 / 2}(\Omega)$ solution of (A.2) such that

$$
\|y\|_{H^{s+1 / 2}(\Omega)} \leq C(M)\|\eta\|_{H^{s}(\Gamma)} .
$$

Moreover, if $0 \leq s \leq 1, \partial_{\nu} y \in H^{s-1}(\Gamma)$ and

$$
\left\|\partial_{\nu} y\right\|_{H^{s-1}(\Gamma)} \leq C(M)\|\eta\|_{H^{s}(\Gamma)} .
$$

Proof. Let us start with $s=1$. Let $y \in H^{1}(\Omega)$ be the solution to equation (A.2). We can write $y=y_{0}+y_{1}$, where $y_{0}$ is the solution to

$$
-\Delta y_{0}=0 \text { in } \Omega, \quad y_{0}=\eta \text { on } \Gamma,
$$

and $y_{1}$ is the solution to

$$
-\Delta y_{1}=-a_{0} y \text { in } \Omega, \quad y_{1}=0 \text { on } \Gamma .
$$

From Jerison and Kenig [14], Theorem 5.6 and Corollary 5.7, it follows that

$$
\left\|y_{0}\right\|_{H^{3 / 2}(\Omega)}+\left\|\partial_{\nu} y_{0}\right\|_{L^{2}(\Gamma)} \leq C\|\eta\|_{H^{1}(\Gamma)} .
$$

Since $-a_{0} y$ belongs to $L^{2}(\Omega)$, from [11] it follows that

$$
\left\|y_{1}\right\|_{H^{2}(\Omega)}+\left\|\left.y_{1}\right|_{\Gamma}\right\|_{H^{1}(\Gamma)} \leq C\left\|a_{0} y\right\|_{L^{2}(\Omega)} \leq C(M)\|\eta\|_{H^{1}(\Gamma)} .
$$

With Lemma A.1, we finally obtain

$$
\left\|\partial_{\nu} y_{1}\right\|_{L^{2}(\Gamma)} \leq C(M)\|\eta\|_{H^{1}(\Gamma)} .
$$

The proof for the case $s=1$ is complete.

Consider now the case when $s=0$. We look for a pair $(y, \chi) \in H^{1 / 2}(\Omega) \times H^{-1}(\Gamma)$ solution to problem (A.16). Let us first show uniqueness. If $(y, \chi) \in H^{1 / 2}(\Omega) \times H^{-1}(\Gamma)$ is a solution to (A.16) for $\eta=0$, taking $g=y$ and $v=0$ we obtain

$$
\int_{\Omega} y^{2} \mathrm{~d} x=0
$$

and thus $y=0$ on $\Omega$. Next we have

$$
\langle\chi, v\rangle_{H^{-1}(\Gamma), H^{1}(\Gamma)}=0 \quad \forall v \in H^{1}(\Gamma)
$$

and hence $\chi=0$.

The existence is obtained by an approximation process. Let $\eta \in L^{2}(\Gamma)$ and let $\eta_{k} \in H^{1}(\Gamma)$ be a sequence such that $\eta_{k} \rightarrow \eta \in L^{2}(\Gamma)$. For each $k$ let $y_{k}$ be the unique solution of (A.2) corresponding to $\eta_{k}$. It is clear that 
$\left(y_{k}, \partial_{\nu} y_{k}\right) \in H^{3 / 2}(\Omega) \times L^{2}(\Gamma)$ is the unique solution of (A.16) for $\eta_{k}$. Take $v=0, g \in L^{2}(\Omega)$ and $z \in H^{2}(\Omega)$ as is defined in (A.16), we can write

$$
\begin{gathered}
\left\|y_{k}\right\|_{H^{1 / 2}(\Omega)}=\sup _{\|g\|_{\left(H^{1 / 2}(\Omega)\right)^{\prime}}=1}\left\langle y_{k}, g\right\rangle_{H^{1 / 2}(\Omega),\left(H^{1 / 2}(\Omega)\right)^{\prime}}=\sup _{\|g\|_{\left(H^{1 / 2}(\Omega)\right)^{\prime}}} \int_{\Omega} y_{k} g \mathrm{~d} x \leq \\
\sup _{\|g\|_{\left(H^{1 / 2}(\Omega)\right)^{\prime}}=1}\left|\int_{\Gamma} \partial_{\nu} z \eta_{k} \mathrm{~d} \sigma(x)\right| \leq \sup _{\|g\|_{\left(H^{1 / 2}(\Omega)\right)^{\prime}}=1}\left\|\partial_{\nu} z\right\|_{L^{2}(\Gamma)}\left\|\eta_{k}\right\|_{L^{2}(\Gamma)} \\
\leq \sup _{\|g\|_{\left(H^{1 / 2}(\Omega)\right)^{\prime}}=1} C(M)\|g\|_{\left(H^{1 / 2}(\Omega)\right)^{\prime}}\left\|\eta_{k}\right\|_{L^{2}(\Gamma)} \leq C(M)\left\|\eta_{k}\right\|_{L^{2}(\Gamma)},
\end{gathered}
$$

where we have used estimate (A.5). In the same way we also obtain

$$
\left\|y_{k}-y_{\ell}\right\|_{H^{1 / 2}(\Omega)} \leq C(M)\left\|\eta_{k}-\eta_{\ell}\right\|_{L^{2}(\Gamma)} .
$$

To get the estimate on the boundary, let us take $g=0$ and $v \in H^{1}(\Gamma)$. Using the case $s=1$ of this lemma (proved above), we obtain

$$
\begin{aligned}
& \left\|\partial_{\nu} y_{k}\right\|_{H^{-1}(\Gamma)}=\sup _{\|v\|_{H^{1}(\Gamma)}=1}\left\langle\partial_{\nu} y_{k}, v\right\rangle_{H^{-1}(\Gamma), H^{1}(\Gamma)}=\sup _{\|v\|_{H^{1}(\Gamma)}=1} \int_{\Gamma} \partial_{\nu} z \eta_{k} \mathrm{~d} \sigma(x) \\
& \leq \sup _{\|v\|_{H^{1}(\Gamma)}=1}\left\|\partial_{\nu} z\right\|_{L^{2}(\Gamma)}\left\|\eta_{k}\right\|_{L^{2}(\Gamma)} \leq \sup _{\|v\|_{H^{1}(\Gamma)}=1} C(M)\|v\|_{H^{1}(\Gamma)}\left\|\eta_{k}\right\|_{L^{2}(\Gamma)}=C(M)\left\|\eta_{k}\right\|_{L^{2}(\Gamma)} .
\end{aligned}
$$

In the same way we also obtain

$$
\left\|\partial_{\nu} y_{k}-\partial_{\nu} y_{\ell}\right\|_{H^{-1}(\Gamma)} \leq C(M)\left\|\eta_{k}-\eta_{\ell}\right\|_{L^{2}(\Gamma)} .
$$

There then exists a pair $(y, \chi) \in H^{1 / 2}(\Omega) \times H^{-1}(\Gamma)$ such that $\left(y_{k}, \partial_{\nu} y_{k}\right) \rightarrow(y, \chi)$ in $H^{1 / 2}(\Omega) \times H^{-1}(\Gamma)$. Taking the limit in the sequence of equations satisfied by $\left(y_{k}, \partial_{\nu} y_{k}\right)$, it is clear that $(y, \chi)$ obeys (A.16). Estimates (A.18) and (A.19) follow from the corresponding ones satisfied by $y_{k}$ and $\partial_{\nu} y_{k}$.

The estimates in the lemma for $0<s<1$ can be obtained by interpolation from the estimates obtained for $s=0$ and the estimates obtained for $s=1$.

Set now $s=-1 / 2$. The proof follows the same lines as the first part of the proof for the case $s=0$. We must use now Lemma A.2 instead of estimate (A.5).

Estimate (A.18) for $-1 / 2<s<0$ is obtained by interpolation.

\section{A.4. Estimates in terms of $\varepsilon$}

For every $\varepsilon>0$, and $-1 \leq s \leq 0$, we define the following Robin-to-Dirichlet operator:

$$
\begin{aligned}
& \Lambda_{\varepsilon}: H^{s}(\Gamma) \rightarrow H^{s+1}(\Gamma) \\
& u \quad \rightarrow \quad \Lambda_{\varepsilon} u=\left.y_{\varepsilon}\right|_{\Gamma},
\end{aligned}
$$

where $y_{\varepsilon}$ is the solution of problem (A.1), making the abuse of notation mentioned above when $-1 \leq s<-1 / 2$. For $\varepsilon=1$ we will set $\Lambda:=\Lambda_{1}$. Estimate (A.14) means that $\Lambda \in \mathcal{L}\left(H^{s}(\Gamma), H^{s+1}(\Gamma)\right.$ ) for all $-1 \leq s \leq 0$.

For $0 \leq s \leq 1$, we define a Dirichlet-to-Robin operator as follows:

$$
\begin{array}{ccc}
T: H^{s+1}(\Gamma) & \rightarrow & H^{s}(\Gamma) \\
\eta & \rightarrow T \eta=\partial_{\nu} y+\eta,
\end{array}
$$

where $\left(y, \partial_{\nu} y\right)$ is the solution of (A.16). 
Estimate (A.19) can be written in the form

$$
\|T \eta\|_{H^{s-1}(\Gamma)} \leq C(M)\|\eta\|_{H^{s}(\Gamma)} \cdot
$$

We have the following relations between $\Lambda_{\varepsilon}$ and $T$.

Lemma A.7. For all $-1 \leq s \leq 0$, all $\varepsilon>0$, all $u \in H^{s}(\Gamma)$ and all $\eta \in H^{s+1}(\Gamma)$, we have

$$
(\varepsilon T+(1-\varepsilon) I) \Lambda_{\varepsilon} u=u,
$$

and

$$
\Lambda_{\varepsilon}(\varepsilon T+(1-\varepsilon) I) \eta=\eta \text {. }
$$

Proof. Let us prove (A.21). Let $u$ belong to $H^{s}(\Gamma)$ and let $y_{\varepsilon}$ be the solution to (A.1). Using the definition of $\Lambda_{\varepsilon}$, we have

$$
(\varepsilon T+(1-\varepsilon) I) \Lambda_{\varepsilon} u=\left.\varepsilon T y_{\varepsilon}\right|_{\Gamma}+\left.(1-\varepsilon) y_{\varepsilon}\right|_{\Gamma}=\varepsilon \partial_{\nu} y_{\varepsilon}+\left.\varepsilon y_{\varepsilon}\right|_{\Gamma}+\left.y_{\varepsilon}\right|_{\Gamma}-\left.\varepsilon y_{\varepsilon}\right|_{\Gamma}=\varepsilon \partial_{\nu} y_{\varepsilon}+\left.y_{\varepsilon}\right|_{\Gamma}=u
$$

Let us prove (A.22). Let $\eta$ belong to $H^{s+1}(\Gamma)$, and let $y \in H^{s+3 / 2}(\Omega)$ be the solution of (A.2). We have

$$
\Lambda_{\varepsilon}(\varepsilon T+(1-\varepsilon) I) \eta=\Lambda_{\varepsilon}\left(\varepsilon \partial_{\nu} y+\varepsilon \eta+\eta-\varepsilon \eta\right)=\Lambda_{\varepsilon}\left(\varepsilon \partial_{\nu} y+\eta\right)=\eta
$$

The proof is complete.

By choosing $\varepsilon=1$, we can state the following results.

Corollary A.8. For all $-1 \leq s \leq 0$ and all $\eta \in H^{s+1}(\Gamma)$,

$$
\Lambda T \eta=\eta
$$

Corollary A.9. For every $u \in L^{2}(\Gamma)$, we have $T \Lambda_{\varepsilon} u=\Lambda_{\varepsilon} T u$.

Proof. With (A.21) and (A.22), we can write:

$$
\varepsilon T \Lambda_{\varepsilon} u+(1-\varepsilon) \Lambda_{\varepsilon} u=u=\varepsilon \Lambda_{\varepsilon} T u+(1-\varepsilon) \Lambda_{\varepsilon} u .
$$

The proof is complete.

Now, we are going to estimate the norm of the operator $\Lambda_{\varepsilon}$.

Proposition A.10. For every $-1 \leq s \leq 1$ and $u \in H^{s}(\Gamma)$

$$
\left\|\Lambda_{\varepsilon} u\right\|_{H^{s}(\Gamma)} \leq C(M)\|u\|_{H^{s}(\Gamma)},
$$

where $C(M)$ is independent of $\varepsilon$ and $s$.

Proof. We first prove the estimate of the proposition for $s=0$. Let $y_{\varepsilon}$ be the solution to (A.1). For all $z \in H^{1}(\Omega)$ we have

$$
\int_{\Omega}\left(\nabla y_{\varepsilon} \nabla z+a_{0} y_{\varepsilon} z\right) \mathrm{d} x+\frac{1}{\varepsilon} \int_{\Gamma} y_{\varepsilon} z \mathrm{~d} \sigma(x)=\frac{1}{\varepsilon} \int_{\Gamma} u z \mathrm{~d} \sigma(x)
$$

We can prove (A.23) for $s=0$ by taking $z=y_{\varepsilon}$, and by taking the equality $\Lambda_{\varepsilon} u=\left.y_{\varepsilon}\right|_{\Gamma}$ into account.

Let us prove (A.23) for $s=1$. With Corollary A.8 and estimate (A.14), we have

$$
\left\|\Lambda_{\varepsilon} u\right\|_{H^{1}(\Gamma)}=\left\|\Lambda T \Lambda_{\varepsilon} u\right\|_{H^{1}(\Gamma)} \leq C(M)\left\|T \Lambda_{\varepsilon} u\right\|_{L^{2}(\Gamma)} .
$$


From Corollary A.9, and with estimate (A.23) for $s=0$, we can write

$$
\left\|T \Lambda_{\varepsilon} u\right\|_{L^{2}(\Gamma)}=\left\|\Lambda_{\varepsilon} T u\right\|_{L^{2}(\Gamma)} \leq C(M)\|T u\|_{L^{2}(\Gamma)} .
$$

Thus, for $s=1$, estimate (A.23) follows from the above inequalities and from (A.20).

For $s=-1$, estimate (A.23) is obtained by duality. Let $v$ be in $H^{1}(\Gamma)$, and set $z_{\varepsilon}=\Lambda_{\varepsilon} v$. From the variational problem (A.12) satisfied by $y_{\varepsilon}$ and $z_{\varepsilon}$, we easily obtain

$$
\left\langle\Lambda_{\varepsilon} u, v\right\rangle_{H^{-1}(\Gamma), H^{1}(\Gamma)}=\left\langle\Lambda_{\varepsilon} u, v\right\rangle_{H^{-1 / 2}(\Gamma), H^{1 / 2}(\Gamma)}=\left\langle u, z_{\varepsilon}\right\rangle_{H^{-1}(\Gamma), H^{1}(\Gamma)} .
$$

So, using estimate (A.23) for $s=1$, one has:

$$
\begin{aligned}
& \left\|\Lambda_{\varepsilon} u\right\|_{H^{-1}(\Gamma)}=\sup _{\|v\|_{H^{1}(\Gamma)}=1}\left\langle\Lambda_{\varepsilon} u, v\right\rangle_{H^{-1}(\Gamma), H^{1}(\Gamma)} \\
& =\sup _{\|v\|_{H^{1}(\Gamma)}=1}\left\langle u, z_{\varepsilon}\right\rangle_{H^{-1}(\Gamma), H^{1}(\Gamma)} \leq C(M)\|u\|_{H^{-1}(\Gamma)} .
\end{aligned}
$$

For $-1<s<1$, estimate (A.23) can be obtained by interpolation.

Proposition A.11. For every $-1 \leq s \leq 0$ and $u \in H^{s}(\Gamma)$

$$
\left\|\Lambda_{\varepsilon} u\right\|_{H^{s+1}(\Gamma)} \leq \frac{C(M)}{\varepsilon}\|u\|_{H^{s}(\Gamma)},
$$

where $C(M)$ is independent of $\varepsilon$ and $s$.

Proof. We first observe that if $u \in L^{2}(\Gamma)$, then we have

$$
\Lambda_{\varepsilon} u=\frac{1}{\varepsilon} \Lambda\left(u-(1-\varepsilon) \Lambda_{\varepsilon} u\right) .
$$

Indeed, if $y_{\varepsilon}$ is the solution of (A.1), one has

$$
\frac{1}{\varepsilon} \Lambda\left(u-(1-\varepsilon) \Lambda_{\varepsilon} u\right)=\frac{1}{\varepsilon} \Lambda\left(\varepsilon \partial_{\nu} y_{\varepsilon}+y_{\varepsilon}-y_{\varepsilon}+\varepsilon y_{\varepsilon}\right)=\frac{1}{\varepsilon} \Lambda\left(\varepsilon\left(\partial_{\nu} y_{\varepsilon}+y_{\varepsilon}\right)\right)=\Lambda\left(\partial_{\nu} y_{\varepsilon}+y_{\varepsilon}\right) .
$$

Identity (A.24) follows from the equality $\Lambda\left(\partial_{\nu} y_{\varepsilon}+y_{\varepsilon}\right)=\left.y_{\varepsilon}\right|_{\Gamma}$.

Using (A.24), the continuity of $\Lambda$ stated in (A.14), and Proposition A.10, we obtain

$$
\begin{aligned}
& \left\|\Lambda_{\varepsilon} u\right\|_{H^{s+1}(\Gamma)}=\left\|\frac{1}{\varepsilon} \Lambda\left(u-(1-\varepsilon) \Lambda_{\varepsilon} u\right)\right\|_{H^{s+1}(\Gamma)} \leq \frac{C(M)}{\varepsilon}\left\|u-(1-\varepsilon) \Lambda_{\varepsilon} u\right\|_{H^{s}(\Gamma)} \\
& \leq \frac{C(M)}{\varepsilon}\left(\|u\|_{H^{s}(\Gamma)}+\left\|\Lambda_{\varepsilon} u\right\|_{H^{s}(\Gamma)}\right) \leq \frac{C(M)(1+C(M))}{\varepsilon}\|u\|_{H^{s}(\Gamma)} .
\end{aligned}
$$

Finally, we are able to prove Theorem 2.2.

Proof of Theorem 2.2. Estimate (2.2) is proved for $(s, t)=(-1,-1),(s, t)=(1,1),(s, t)=(0,1)$ and $(s, t)=$ $(-1,1)$ in Propositions A.10 and A.11. Thus, by interpolation, it is also true for the convex hull of these four points, which is precisely what we need.

From (A.18), it follows that

$$
\left\|y_{\varepsilon}\right\|_{H^{t+1 / 2}(\Omega)} \leq C(M)\left\|\left.y_{\varepsilon}\right|_{\Gamma}\right\|_{H^{t}(\Gamma)},
$$


for all $-1 / 2 \leq t \leq 1$. Thus, with $(2.2)$, we have

$$
\left\|y_{\varepsilon}\right\|_{H^{t+1 / 2}(\Omega)} \leq C(M)\left\|\left.y_{\varepsilon}\right|_{\Gamma}\right\|_{H^{t}(\Gamma)} \leq C \varepsilon^{s-t}\|u\|_{H^{s}(\Gamma)},
$$

for all $-1 / 2 \leq s \leq 1$, and all $s \leq t \leq \min \{1, s+1\}$.

\section{REFERENCES}

[1] J.-J. Alibert and J.-P. Raymond, Boundary control of semilinear elliptic equations with discontinuous leading coefficients and unbounded controls. Numer. Funct. Anal. Optim. 18 (1997) 235-250.

[2] F. Ben Belgacem, H. El Fekih and H. Metoui, Singular perturbation for the Dirichlet boundary control of elliptic problems. ESAIM: M2AN 37 (2003) 833-850.

[3] F. Ben Belgacem, H. El Fekih and J.-P. Raymond, A penalized Robin approach for solving a parabolic equation with nonsmooth Dirichlet boundary conditions. Asymptot. Anal. 34 (2003) 121-136.

[4] E. Casas and M. Mateos, Error estimates for the numerical approximation of Neumann control problems. Comput. Optim. Appl. 39 (2008) 265-295.

[5] E. Casas and J.-P. Raymond, Error estimates for the numerical approximation of Dirichlet boundary control for semilinear elliptic equations. SIAM J. Contr. Opt. 45 (2006) 1586-1611 (electronic).

[6] E. Casas and J.-P. Raymond, The stability in $W^{s, p}(\Gamma)$ spaces of $L^{2}$-projections on some convex sets. Numer. Funct. Anal. Optim. 27 (2006) 117-137.

[7] E. Casas, M. Mateos and F. Tröltzsch, Error estimates for the numerical approximation of boundary semilinear elliptic control problems. Comput. Optim. Appl. 31 (2005) 193-219.

[8] P.G. Ciarlet, Basic error estimates for elliptic problems, in Handbook of Numerical Analysis II, North-Holland, Amsterdam (1991) $17-351$.

[9] M. Costabel and M. Dauge, A singularly perturbed mixed boundary value problem. Comm. Partial Diff. Eq. 21 (1996) 1919-1949.

[10] Z. Ding, A proof of the trace theorem of Sobolev spaces on Lipschitz domains. Proc. Amer. Math. Soc. 124 (1996) 591-600.

[11] P. Grisvard, Elliptic Problems in Nonsmooth Domains. Pitman, Boston (1985).

[12] L.S. Hou and S.S. Ravindran, A penalized Neumann control approach for solving an optimal Dirichlet control problem for the Navier-Stokes equations. SIAM J. Contr. Opt. 36 (1998) 1795-1814 (electronic).

[13] D. Jerison and C. Kenig, The Neumann problem on Lipschitz domains. Bull. Amer. Math. Soc. (N.S.) 4 (1981) $203-207$.

[14] D. Jerison and C.E. Kenig, The inhomogeneous Dirichlet problem in Lipschitz domains. J. Funct. Anal. 130 (1995) $161-219$.

[15] C.V. Pao, Nonlinear parabolic and elliptic equations. Plenum Press, New York (1992).

[16] J.-P. Raymond, Stokes and Navier-Stokes equations with nonhomogeneous conditions. Ann. Inst. H. Poincaré Anal. Non Linéaire 24 (2007) 921-951.

[17] G. Stampacchia, Le problème de Dirichlet pour les équations elliptiques du second ordre à coefficients discontinus. Ann. Inst. Fourier (Grenoble) 15 (1965) 189-258. 